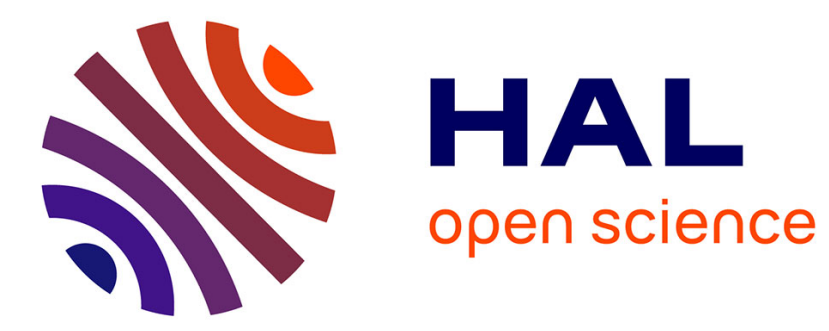

\title{
Superficial segregation in nanoparticles from facets to infinite surfaces
}

\author{
F. Lequien, J. Creuze, F. Berthier, B. Legrand
}

\section{To cite this version:}

F. Lequien, J. Creuze, F. Berthier, B. Legrand. Superficial segregation in nanoparticles from facets to infinite surfaces. Journal of Chemical Physics, 2006, 125 (9), pp.094707. 10.1063/1.2245804 . cea-02356034

\section{HAL Id: cea-02356034 https://hal-cea.archives-ouvertes.fr/cea-02356034}

Submitted on 2 Dec 2019

HAL is a multi-disciplinary open access archive for the deposit and dissemination of scientific research documents, whether they are published or not. The documents may come from teaching and research institutions in France or abroad, or from public or private research centers.
L'archive ouverte pluridisciplinaire HAL, est destinée au dépôt et à la diffusion de documents scientifiques de niveau recherche, publiés ou non, émanant des établissements d'enseignement et de recherche français ou étrangers, des laboratoires publics ou privés. 


\title{
Superficial segregation in nanoparticles: from facets to infinite surfaces.
}

\author{
F. Lequien, J. Creuze*, and F. Berthier \\ LEMHE/ICMMO, Bât. 410, Université Paris XI, F91405 Orsay Cedex, France \\ B. Legrand \\ SRMP-DMN, CEA Saclay, F91191 Gif-sur-Yvette Cedex, France
}

\begin{abstract}
We compare the superficial segregations of the $\mathrm{Cu}-\mathrm{Ag}$ system for a nanoparticle and for surfaces that are structurally equivalent to each of its facet. Based on a lattice-gas model and within a meanfield formalism, we derive segregation isotherms at various temperatures in the canonical ensemble, i.e., for a given overall solute concentration, and in the semi-grand canonical ensemble, i.e., for a given bulk solute concentration. If both processes are very similar for high temperatures, they differ substancially at lower temperatures. Due to the finite-size effect and the indirect coupling between facets and edges, the relative position of the phase transitions of the facets and the corresponding surfaces are inversed when displayed as function of the solute bulk concentration. Moreover, we show that working in the semi-grand canonical ensemble is a much more efficient way to study this phenomenon, although nanoparticles are 'canonical' objects in essence.
\end{abstract}

\footnotetext{
* Corresponding author: Jerome.Creuze@lemhe.u-psud.fr
} 


\section{INTRODUCTION}

In binary alloys, the repartition of the constituents near crystallographical defects (point defects, dislocations, surfaces and interfaces) differs from the bulk one. The magnitude of this segregation phenomenon, intensively studied for surfaces and grain boundaries [1-4], increases for systems with large surface/bulk ratios, e.g., thin films or nanoparticles. Both the extensive use of mono- and bimetallic nanoparticles in heterogeneous catalysis [5] and the optimisation of technological applications that depend mainly on surface properties has motivated numerous studies about the thermodynamics of finite-size systems, and more especially the segregation in bimetallic nanoparticles [6-23]. Due to strong limitations in current experimental tools, most of the studies are based on theoretical approaches and focus on the nanoparticle superficial composition versus temperature and overall composition. More recently, Polak and Rubinovich have shown that an intercluster separation coupled with an intracluster surface segregation may occur in binary and ternary alloy nanoparticles at 'magic number' compositions [21].

In this work, we compare the superficial segregations of nanoparticles with semi-infinite alloys, to determine the respective roles of the crystallographic orientation of the surface and the finite-size effect. Besides the enrichment amplitude characterized by the segregation isotherms, i.e., the evolution of the superficial composition with the bulk one at a given temperature, we are also interested in the superficial phase transitions that may occur for systems with a tendency to phase separate in the bulk. Previous studies about superficial segregation for clustering-type solid solutions have predicted transitions evocating a surface miscibility gap using a regular solution model [24]. More detailed studies revealed the existence of layering transitions [25] that can be related to a wetting phenomenon [26]. This kind of transition is characterized by a sharp increase of the surface concentration of the solute atom as its bulk concentration increases or the temperature decreases [27, 28]. For nanoparticles surfaces, two factors may change these phase transitions: the finite-size effects and the couplings between the different (inequivalent) sites of the external shell (vertices, edges and facets). The objective of this present work is to study the respective role of these two factors on the distinctive features of the segregation isotherms for nanoparticles.

We choose the $\mathrm{Cu}-\mathrm{Ag}$ system as its superficial segregation behavior has been extensively studied [2, 29]. We shall consider it as a general model of alloys that exhibit a strong tendency 
to phase separate in the bulk. Then, we need an energetic model and a statistical approach (mean-field approximation (MFA) [2] or Monte Carlo simulations (MC) [30]) to minimize the free energy with respect to local concentrations. To describe the atomic interactions, we choose a simple energetic model that consists in considering a rigid lattice for which the energy is expressed as a sum of pair interactions, known as 'lattice-gas model' or 'Ising model'. Note that a rather similar approach has been used recently by Polak and Rubinovich [21]. They developped an analytical model taking into account interatomic correlations and using pair interactions based on coordination-dependent site energies [9, 16]. For a system that tends to phase separate in the bulk, the comparison between segregation isotherms obtained within a MFA and those obtained by MC simulations shows that interatomic correlations mainly lowers the critical temperature without changing the overall segregation behavior [31].

The paper is organized as follows: in Sect. II, we present the nanoparticle and the models. In Sect. III, segregation isotherms for the infinite surfaces are briefly recalled and then compared with the nanoparticle ones at high temperature both within a MFA and MC simulations. In Sect. IV, we study segregation isotherms at low temperature and we detail the differences between infinite surfaces and the nanoparticle within a MFA. We draw our conclusions in Sect. V.

\section{MODELS}

\section{A. Cuboctahedron}

Due to their finite size, nanoparticles adopt crystallographic structures that depend on the total number of atoms [32]. For face-centered cubic metals, while the icosahedron is more stable than the cuboctahedron (which relies on a fcc lattice) at small sizes, the cuboctahedron becomes the most stable structure for a specific size that depends on the metal [33, 34]. Here, we chose a 3871 atoms nanoparticle in a cuboctahedral shape, this structure being more stable than the icosahedron both for pure copper and silver nanoparticles. This cluster is constituted of 12 vertices, 24 edges, 6 square (100) facets and 8 (111) triangular facets (see Fig. 1). We recall that if the truncated octahedron (Wulff polyhedron) is more stable than the cuboctahedron, both structures are based on the fcc lattice, the main difference 
being given by the (001)facet/(111)facet area ratio $(>1$ for the cuboctahedron and $<1$ for the truncated octahedron).

We generate the cuboctahedron by a successive packing of atoms as follows: the central atom surrounded by its twelve nearest neighbors of the fcc structure defines the nanoparticle of order $n=1$. Adding a new shell of nearest neighbors gives the nanoparticle of order $n=2$ and so on. The 3871 atoms nanoparticle corresponds to $n=10$. We will focus only on its superficial composition as regard to the composition of the inner cluster of size $n-2$, to be called the core in the following. The composition of the first underlying shell is not fixed at the core one a priori. Four types of sites (classes) are distinguished on the external shell: the vertices $(\mathrm{V})$, the edges $(\mathrm{E})$, the (001) facets $[(001) \mathrm{F}]$ and the (111) facets [(111)F]. In contrast with surfaces of semi-infinite crystals, inside a class, the type of bonds for an atom varies: at the center of a (111) facet, an atom has six nearest neighbors within the facet, whereas close to the edges an atom may own two (or four) bonds with atoms of this same facet (intra-class bonds) and four (or two) bonds with atoms of the neighboring edge(s) (inter-class bonds). This leads to consider the total number of inter-class bonds, $N_{p, q}$, and intra-class bonds, $N_{p, p}$, between $p$ and $q$ classes of sites to study nanoparticles within a mean-field formalism (see table I) [7]. Moreover, we can compare the coordination numbers obtained for facets, $Z_{p, q}=\frac{N_{p, q}}{N_{p}}, N_{p}$ being the number of sites of the $p$-class, with the coordination numbers of surfaces with the same crystallographic orientation (table II). Contrary to nanoparticles, surfaces may be considered as a packing of atomic planes with the same number of atoms: $Z_{p, q}=Z_{q, p}$. Moreover, edges (resp. vertices) and sites of a (110) surface (resp. an isolated adatom on a (110) surface, noted (110)ads in the following) share the same coordination number. This allows one to define equivalent infinite surfaces (EIS).

\section{B. Lattice-gas model}

Within a lattice-gas model in which only nearest-neighbor pair interactions are taken into account, a binary alloy $\mathrm{A}_{c} \mathrm{~B}_{1-c}$ can be described by the Ising Hamiltonian [35]:

$$
H=\frac{1}{2} \sum_{I, J} \sum_{i, j \neq i} p_{i}^{I} p_{j}^{J} \epsilon^{I J}
$$

where $\epsilon^{I J}$ is the interaction energy between an atom of type $I$ at the site $i$ and an atom of type $J$ at the site $j[(I, J)=(\mathrm{A}, \mathrm{B})], i$ and $j$ being in nearest-neighbor position. $p_{i}^{I}$ is the 
occupation number that equals $1(0)$ if the site $i$ is (not) occupied by an atom of type $I$. For a binary alloy, $p_{i}^{A}=1-p_{i}^{B}$ and we set $p_{i}^{A}=p_{i}$, which leads to:

$$
H=H_{0}+\sum_{i, j \neq i} p_{i} p_{j} \epsilon+\sum_{i} \sum_{j \neq i} p_{i}(\tau-\epsilon)
$$

$i$ and $j$ being sites in nearest-neighbors positions. $\tau=\frac{1}{2}\left(\epsilon^{A A}-\epsilon^{B B}\right)$ is proportional to the difference between cohesive energies of pure metals, $H_{0}=\frac{1}{2} \sum_{i, j \neq i} \epsilon^{B B}$, and $\epsilon=$ $\frac{1}{2}\left(\epsilon^{A A}+\epsilon^{B B}-2 \epsilon^{A B}\right)$ is the energy of alloy pair interaction that characterizes the system tendency to favor homoatomic $(\epsilon<0)$ or heteroatomic $(\epsilon>0)$ pairs.

\section{Mean-field approximation}

The Bragg-Williams approximation (one-site mean-field approximation) consists in neglecting short-range order. Thus, occupations of sites $i$ and $j$ are no more coupled: $<p_{i} p_{j}>=<p_{i}><p_{j}>$. The system is described as an ensemble of $p$ classes of $N_{p}$ sites $i$ with homogeneous concentrations $c_{p}$ such as $\left\langle p_{i}\right\rangle=c_{p}$. Internal energy can then be written as:

$$
<H>=H_{0}+(\tau-\epsilon) \sum_{p} Z_{p} N_{p} c_{p}+\epsilon \sum_{p, q} N_{p, q} c_{p} c_{q},
$$

where $Z_{p}=\sum_{q} Z_{p, q}$ is the coordination number of the $p$-class sites and $N_{p, q}$ numbers of the nearest-neighbor bonds between sites of classes $p$ and $q$. Within the semi-grand canonical ensemble, the free energy is expressed as $F=<H>-T S-\Delta \mu \sum_{p} N_{p} c_{p}$, where $T$ is the temperature, $\Delta \mu=\mu_{A}-\mu_{B}$ is the difference in chemical potentials of pure metals and $S$ is the configurational entropy within the Bragg-Williams approximation [36]. We can then rewrite $F$ as:

$$
\begin{aligned}
F & =H_{0}+(\tau-\epsilon) \sum_{p} Z_{p} N_{p} c_{p}+\epsilon \sum_{p, q} N_{p, q} c_{p} c_{q} \\
& +k_{B} T \sum_{p} N_{p}\left[c_{p} \ln c_{p}+\left(1-c_{p}\right) \ln \left(1-c_{p}\right)\right]-\Delta \mu \sum_{p} N_{p} c_{p},
\end{aligned}
$$

where $k_{B}$ is the Boltzmann constant. The equilibrium profile is obtained for $\left(\frac{\partial F}{N_{p} \partial c_{p}}\right)_{c_{q}(q \neq p)}$ $=0 \forall p$, leading to:

$$
\frac{c_{p}}{1-c_{p}}=\exp \left(-\frac{\Delta H_{p}^{p e r m}-\Delta \mu}{k_{B} T}\right)
$$

with

$$
\Delta H_{p}^{p e r m}=Z_{p}(\tau-\epsilon)+2 \epsilon \sum_{q} Z_{p, q} c_{q}
$$


$\Delta H_{p}^{p e r m}$ is the permutation energy and corresponds to the energy balance due to the transformation of an atom of type $B$ in an atom of type $A$ on a $p$-class site. Note that $\Delta H_{p}^{p e r m}$ is the sum of two terms. The first one,

$$
\Delta H_{p}^{\text {perm,imp }}=Z_{p}(\tau-\epsilon)
$$

corresponds to the infinitely dilute limit $\left(c_{q} \rightarrow 0, \forall q\right)$ and is proportional to the $p$-class coordination number. The second one,

$$
\Delta H_{p}^{\text {perm, alloy }}=2 \epsilon \sum_{q} Z_{p, q} c_{q}
$$

corresponds to the alloying term and accounts for interactions between segregated atoms. For superficial segration in semi-infinite systems, it is more common to write Eq. (5) by replacing $\Delta \mu$ with its expression as a function of the bulk concentration $c_{b u l k}$ :

$$
\Delta \mu=k_{B} T \ln \left(\frac{c_{b u l k}}{1-c_{b u l k}}\right)+2 \epsilon Z_{b u l k} c_{b u l k}+Z_{b u l k}(\tau-\epsilon)
$$

where $Z_{b u l k}$ is the coordination number for the fcc structure. This leads to the well-known segregation equation [2]:

$$
\frac{c_{p}}{1-c_{p}}=\frac{c_{\text {bulk }}}{1-c_{\text {bulk }}} \exp \left(-\frac{\Delta H_{p}^{\text {seg }}}{k_{B} T}\right),
$$

where the segregation energy $\Delta H_{p}^{\text {seg }}$ is the energy variation when an $A$ solute atom in the bulk is exchanged with a $B$ solvent atom in a $p$-class site. This exchange can be separated in two elementary permutations: $B \rightarrow A$ in the $p$-class, corresponding to $\Delta H_{p}^{\text {perm }}$, and $A \rightarrow B$ in the bulk, corresponding to $-\Delta H_{b u l k}^{\text {perm }}$. Therefore,

$$
\Delta H_{p}^{\text {seg }}=\Delta H_{p}^{\text {perm }}-\Delta H_{b u l k}^{\text {perm }}
$$

If Eq. (10) is more commonly used than Eq. (5), it requires a bulk reference which may be a problem for nanoparticles. Actually, evolutions of Ag core concentration, $c_{\text {core }}$, and of $c_{b u l k}$ as a function of $\Delta \mu$ may be different for very small nanoparticles, due to couplings of the core with the external shell. Note that using the semi-grand canonical ensemble allows the connection with the recent study on cluster systems by Polak and Rubinovich [21], which leads to intercluster separation. From this point of view, one can note that an isolated nanoparticle constitutes already a vertices, edges and facets system, each element 
of a given class of sites being able to exchange constituent atoms with an other element of the same class.

In the following, the results have been obtained by solving numerically Eqs. (5), $c_{\text {bulk }}$ and $c_{\text {core }}$ being evaluated from Eq. (9) later on (the nanoparticle of order $n=10$ is sufficiently large to recover the properties of a fcc bulk in its core region). This represents 6 equations for the nanoparticle ( 4 for the external shell, 1 for the first underlying shell and $c_{\text {core }}$ for the second underlying shell) and 3 equations for the infinite surfaces ( 1 for the surface plane, 1 for the first underlayer and $c_{b u l k}$ for the second underlayer).

\section{Energetic parameters}

The energetic model described above depends on two thermodynamical quantities: the alloying pair interaction $\epsilon$ and the permutation energy in the infinitely dilute limit $\Delta H_{p}^{p e r m, i m p}$, the latter depending on $\epsilon$ and $\tau$ [see Eq. (7)]. The main properties of the $\mathrm{Cu}-\mathrm{Ag}$ system are then reproduced by taking $\tau=46 \mathrm{meV}$ and $\epsilon=-30 \mathrm{meV}$ [29]. At this stage, two remarks can be made:

- since $\Delta H_{p}^{p e r m, i m p}$ depends linearly on the coordination number [see Eq. (7)], discarding bonds on a site shifts its isotherm towards lower $\Delta \mu$. This means that the segregation will occur preferentially on vertices, then on edges, on (100) facets and finally on (111) facets.

- The permutation energies in the infinitely dilute limit are identical for a site of a given class of the nanoparticle and for equivalent infinite surfaces, see Table II.

Moreover, note that there is no segregation on the sites located under the shell (or under the surface plane) in the infinitely dilute limit since they have the same coordination number as in the bulk. This means that the segregation isotherms on these sites are slightly influenced by the isotherms of the more external sites. Actually, for a $\mathrm{Cu}$ nanoparticle with an $\mathrm{Ag}$ surface shell, the alloying term in $\Delta H_{p}^{s e g}$ for the underlying shell is $2 \epsilon\left(Z_{5, V}+Z_{5, E}+Z_{5,(001) F}+Z_{5,(111) F}\right)=-120 \mathrm{meV}$, this value being small when compared with the segregation energies in the infinitely dilute limit of the different classes of sites (see Table III). Therefore, one can expect that the Ag enrichment of the nanoparticle external shell (or the surface plane) will occur for $\Delta \mu$ 's corresponding to an Ag concentration of 
the underlying shell (or plane) almost equal to $c_{\text {core }}$ (or $c_{b u l k}$ ). For superficial segregation, this leads to the well-known monolayer model [29] and Eq. (5) of the surface layer $(p=0)$ becomes in the bulk dilute limit:

$$
\frac{c_{0}}{1-c_{0}}=\exp \left(-\frac{\Delta H_{0}^{\text {perm,imp }}+2 \epsilon Z_{00} c_{0}-\Delta \mu}{k_{B} T}\right) .
$$

Equation (12) is simply the well-known Fowler-Guggenheim equation, which leads to the existence of a first-order phase transition on the surface plane [37]. Below a critical temperature, $T_{c}^{0}$, the isotherm of the surface plane presents a symmetrical Van der Waals loop. Extension to the nanoparticle external shell with its four classes of sites is straightforward and the $p$-class critical temperature of phase transition, $T_{c}^{p}$, simply corresponds to the critical temperature of a system characterized by a coordination number $Z_{p p}$ :

$$
T_{c}^{p}=-\frac{Z_{p p} \epsilon}{2 k_{B}} .
$$

The values of $T_{c}^{p}$ are given in Table III for the different classes of the external shell and for the equivalent infinite surfaces. This allows one to define a high temperature regime, $T>1044 \mathrm{~K}$, for which no superficial phase transition occurs, and a low temperature regime, $T<348 \mathrm{~K}$, for which phase transitions occur for all $p$-classes and all surface orientations (at least those with $Z_{p p} \neq 0$ ). Finally, Eq. (12) can be rewritten as a function of $T_{c}^{p}$ :

$$
\frac{c_{p}}{1-c_{p}}=\exp \left(-\frac{\Delta H_{p}^{\text {perm }, i m p}-\Delta \mu}{k_{B} T}\right) \exp \left(4 \frac{T_{c}^{p}}{T} c_{p}\right) \text {. }
$$

\section{SEGREGATION AT HIGH TEMPERATURE}

At high temperature, local correlations become negligible and first-order phase transitions are no more expected. At $T=1100 \mathrm{~K}$, the nanoparticle external shell and the equivalent infinite surfaces are situated in the total miscibility domain of their 2D phase diagrams, whereas the 3D phase diagrams of the nanoparticle core and semi-infinite bulk still exhibit a miscibility gap. We can therefore expect that the monolayer model described in the previous section will apply.

\section{A. Segregation isotherms within the MFA formalism}

Figure 2a shows the evolution of the concentration for the different classes of the nanoparticle external shell (V, E, (001)F and (111)F) as a function of $\Delta \mu$. The segregation isotherms 
are continuous and do not present any Van der Waals loop. A more detailed analysis of the segregation isotherms shows that for low values of $\Delta \mu, c_{V}>c_{E}>c_{(001) F}>c_{(111) F}$ as expected from the linear dependence of $\Delta H_{p}^{p e r m, i m p}$ with the coordination number. A similar site-specific segregation was already obtained in one of the first Monte Carlo studies of bimetallic clusters [38]. Moreover, Eq. (14) shows that the isotherm slope at $c_{p}=0.5$ is proportional to the critical temperature (or the intra-site bond number). This behavior is well reproduced and leads to a hierarchy inversion between (001) and (111) facets for large $\Delta \mu$ (Fig. 2a). We will discuss the characteristics of the intersection point into the next section.

Figure $2 \mathrm{~b}$ depicts the segregation isotherms as often encountered in the litterature, i.e., the concentration evolutions for the different classes of sites as a function of the total number of silver atoms, $N_{A g}=\sum_{p} N_{p} c_{p}+N_{\text {core }} c_{\text {core }}, c_{\text {core }}$ being evaluated from Eq. (9). This representation permits to connect with experiments. For a more detailed interpretation of the equilibrium structure of the nanoparticle, we have also added the isotherm of the core concentration. For $N_{A g}>1500$, the nanoparticle external shell is almost pure in silver, whereas the nanoparticle core is almost pure in copper. This configuration is the so-called core-shell structure and the present study shows that it can be obtained even at high temperature.

\section{B. Facets versus equivalent infinite surfaces}

We can wonder whether the behavior of the nanoparticle external shell is merely the sum of the EIS behaviors. Moreover, does the hierarchy inversion between (001) and (111) facets (see Fig. 2) also seen for the semi-infinite alloy surfaces? In Figure 3, we compare the segregation isotherms of each nanoparticle class of sites and each EIS. For vertices and (001) facets, describing in terms of EIS is very satisfactory (Figs. 3a and 3c). For edges and (111) facets, we observe a shift in $\Delta \mu$ and a difference in the slopes for the (111) case (Figs. $3 \mathrm{~b}$ and $3 \mathrm{~d})$. These differences can be explained as follows:

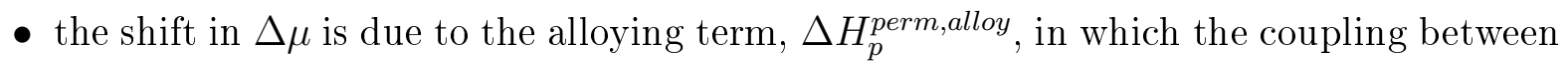
segregating species is different when comparing the nanoparticle and the equivalent infinite surfaces. For the latter, it is only due to the intra-class interactions, segregation on the underlying planes being almost negligible. For the nanoparticle, $\Delta H_{p}^{\text {perm,alloy }}$ depends also on the inter-class interactions. Therefore, silver enrichment of vertices 
induces an increase of $\Delta H_{E}^{\text {perm }}$ for edges, in absolute value, which results in a shift of the edge isotherm towards lower $\Delta \mu$ (fig. 3d). The (001) and (111) facets isotherms are also shifted towards lower $\Delta \mu$, the (001) isotherm being however much less shifted than the (111) one. This is mainly due to the difference in coordination numbers between edges and the different facets: $Z_{(001) F, E}<Z_{(111) F, E}$ (see Table II), leading to a less important coupling between edges and (001) facets than with (111) facets.

- The difference in the slopes for the (111) facet and its equivalent infinite surface is due to the difference in the intra-class coordination number for the facet $\left(Z_{\text {intra }}=4.7\right)$ and for the (111) surface $\left(Z_{\text {intra }}=6\right.$, see Table II). Note that such a difference exists also for the (001) orientation, but it is very weak, leading to slopes that are very similar for this specific orientation $\left(Z_{\text {intra }}=4\right.$ for the equivalent infinite surface and $Z_{\text {intra }}=3.6$ for the facet).

Figure 4 shows a reconstitution of the nanoparticle external shell with the four EIS segregation isotherms. The overall behavior follows the same trend. More precisely, segregation hierarchy between (001) and (111) surfaces as a function of $\Delta \mu$ also inverses. Thus, this inversion is not only related to the facets coupling via edges but it is also due to the facets crystallography. The more important the number of broken bonds for a surface, the higher (in absolute value) its segregation energy but the smoother the slope of its segregation isotherm, as $Z_{\text {intra }}$ being smaller. So there exists a subtle balance between the infinitely dilute term and the alloying term of the segregation enthalpy that can lead to such an inversion of the segregation hierarchy between two surfaces of different crystallographical orientations. Experimentally, studies about the anisotropy of superficial segregation using surfaces with different crystallographic orientations and the same experimental procedures (temperature and bulk concentration) are still not much documented [39-42].

\section{MFA versus Monte Carlo simulations}

Beyond the MFA framework we shall now check the assumption of homogeneous concentrations in the different classes of sites. We therefore perform Monte Carlo simulations in the semi-grand canonical ensemble with the same Hamiltonian. MC segregation isotherms are averaged over all sites of a same class once the equilibrium is reached. The comparison 
between MFA and MC results (see Fig. 2) only reveals a weak deviation for the segregation isotherms of the (001) and (111) facets for $N_{A g}>600$, due to two reasons:

- for $600<N_{A g}<1200$, it derives from the difference in critical temperatures between both methods. Indeed, it is well-known that the MFA overestimates the critical temperatures of first-order phase transitions [43]. Thus, isotherms obtained within the MFA formalism are steeper than the ones obtained by MC simulations at the same temperature $\left(T>T_{c}\right)$, see Figure $2 \mathrm{a}$. This is well illustrated with the isotherms for the (111) facet (corresponding to the highest $T_{c}$ ) for which the deviation can be described as an overestimation of $c_{(111) F}$ within the MFA when $\Delta \mu>\Delta \mu_{c}$.

- For $N_{A g}>1200$, it derives from the difference in the evaluation of $N_{A g}$ by the two methods. Within the MC simulations, the total number of silver atoms is averaged over all sites of the nanoparticle during the run whereas within the MFA, we assume that the Ag core concentration is recovered on the second layer under the external shell. In fact, for systems with a tendency to phase separate $(\epsilon<0)$, the concentration profile is monotonous as a function of the distance to the surface plane [2], leading to a slight silver enrichment of the underlying planes far from the surface. Therefore, the MFA leads to a small underestimation of $N_{A g}$, resulting in a weak shift of the segregation isotherms towards lower $N_{A g}$.

However, the agreement between both methods is satisfactory, which allows the use of the MFA formalism to study superficial segregation in nanoparticles.

\section{SEGREGATION ISOTHERMS AT LOW TEMPERATURE WITHIN THE MFA FORMALISM}

We consider now the segregation isotherms obtained at low temperatures, i.e., $T=300$ K. This temperature is lower than the smallest critical temperature of first-order phase transition in the MFA (see Eq. 13 and Table III), corresponding to the edges one. Therefore, at this temperature and within the MFA formalism, we can predict that first-order phase transitions will occur for the nanoparticle edges and facets. To compare the nanoparticle behavior with the surfaces one, we first present the results obtained for the surfaces of semi-infinite alloys, then for the nanoparticle. 


\section{A. Segregation isotherms for the equivalent infinite surfaces}

The superficial segregation isotherms obtained at $T=300 \mathrm{~K}$ for the four equivalent infinite surfaces are shown Figure 5a. They exhibit Van der Waals loops that are characteristic of first-order phase transitions, except for the segregation isotherm of an isolated adatom on a (110) plane. This result is not surprising, such transitions being related to the intraclass coupling term of the permutation energy [see Eq. (8)]. An isolated adatom having no intra-class coupling, its segregation isotherm is continuous at any temperature. Note also that the other three isotherms cross at the same point. Using Eq. (5) and searching for the intersection points of the different isotherms within the monolayer model [Eq. (14)], we find that a unique intersection point exists, with the following characteristics:

$$
c_{\text {surf }}^{\text {int }}=\frac{1}{4}\left(1-\frac{\tau}{\epsilon}\right) \text { and } \Delta \mu_{\text {surf }}^{\text {int }}=6(\tau-\epsilon),
$$

leading to $c_{\text {surf }}^{\text {int }}=0.63$ and $\Delta \mu_{\text {surf }}^{\text {int }}=0.456 \mathrm{eV}$, in total agreement with the numerical results. This result is obtained only if the monolayer model can be used, thus at sufficiently low temperatures to prevent segregation on the first underlayer. Conversely, at high temperatures, e.g. for $T=1100 \mathrm{~K}$, Eq. (15) is no more valid due to the coupling with the underlying planes in the alloying term of the permutation energy, see Eq. (8).

To highlight these phase transitions, we present on Fig. 5b only the stable part of the same segregation isotherms, obtained by selecting the state with the lowest free energy for each $\Delta \mu$. Therefore, Van der Waals loops (that contain the metastable and unstable parts of the isotherms) are replaced by discontinuous jumps in concentration at $\Delta \mu_{c}^{p}$ from values near 0 to values near 1 for $T<<T_{c}^{p}$. If the nanoparticle follows a similar behavior as its equivalent infinite surfaces, we should expect in MFA a progressive Ag enrichment on vertices followed by successive first-order phase transitions with the sequence edges/(100) facet/(111) facet, as a function of $\Delta \mu$.

\section{B. Segregation isotherms for the nanoparticle}

Figure 6 presents the segregation isotherms obtained at $T=300 \mathrm{~K}$ for the nanoparticle. As expected, the vertices isotherm has no phase transition, contrary to those for the edges and the facets. The edge isotherm exhibits a main Van der Waals loop followed by secondary companion loops due to transitions for the (001) and (111) facets. The intersection point 
between (001)F and (111)F isotherms strongly differs from the previous case, due to the coupling of the facets with the edges. Its characteristics, as a function of the order, $n$, of the nanoparticle is given by:

$$
(n-5) c_{F_{1}, F_{2}}^{i n t}=\frac{n-1}{4}\left(1-\frac{\tau}{\epsilon}\right)-4 c_{E}\left(c_{F_{1}, F_{2}}^{i n t}\right)
$$

where $c_{F_{1}, F_{2}}^{i n t}$ is the intersection point between the two facet isotherms and $c_{E}$ is the edge concentration at the corresponding $\Delta \mu$. If we assume that $c_{E}\left(c_{F_{1}, F_{2}}^{i n t}\right) \approx 1$, we find $c_{F_{1}, F_{2}}^{i n t} \approx 0.33$, in very good agreement with the numerical result. Note that for high-order nanoparticles, i.e. $n \rightarrow \infty$, Eqs. (16) resumes to Eq. (15). Thus, the change in the intersection point between the isotherms of the (001) and (111) facets is an illustration of the finite-size effect on the segregation behavior.

As the four classes of sites for nanoparticles are connected, determining the succession of the different phase transitions with $\Delta \mu$ is not as straightforward as for the equivalent infinite surfaces. To solve this problem efficiently, we draw the ground states of the Hamiltonian as a function of $\Delta \mu$ [29], by comparing for each $\Delta \mu$ the internal energies of all the different configurations obtained by filling $0,1,2, \ldots \eta$ classes of sites of the external shell with $\mathrm{Ag}$ atoms:

$$
\begin{gathered}
H_{0}(\Delta \mu)=0 \\
H_{1}^{p}(\Delta \mu)=N_{p} \Delta H_{p}^{p e r m, i m p}+N_{p, p} \epsilon-N_{p} \Delta \mu
\end{gathered}
$$

with $p=V, E,(001) F$ or $(111) F$,

$$
H_{2}^{p q}(\Delta \mu)=N_{p} \Delta H_{p}^{p e r m, i m p}+N_{q} \Delta H_{q}^{p e r m, i m p}+\epsilon\left(N_{p, p}+N_{q, q}+N_{p, q}+N_{q, p}\right)-\left(N_{p}+N_{q}\right) \Delta \mu
$$

with $(p, q)=(V, E),(V,(001) F),(V,(111) F),(E,(001) F),(E,(111) F)$ or $((001) F,(111) F)$. This process is iterated up to the complete filling of the external shell. Among the sixteen different configurations, Figure 7 only depicts those with low free energy within the interval of $\Delta \mu$ where the phase transitions occur. Analysis of the ground states shows that there are four distinct ranges in $\Delta \mu$, each one being characterized by the filling in silver of one additional class of sites as $\Delta \mu$ increases, following the order: $\mathrm{V}, \mathrm{V}+\mathrm{E}, \mathrm{V}+\mathrm{E}+(111) \mathrm{F}$ and finally the entire external shell. This is illustrated on Figure 8a where only stable states of the isotherms are represented. While the MFA formalism predicts a similar behavior for the segregation on nanoparticle vertices and edges with their equivalent infinite surfaces, it 
strongly differs for the two facets. This is due to the coupling between facets and edges that disappears when considering each equivalent infinite surface separately. In fact, there is a range in $\Delta \mu$ for which edges are almost Ag-pure whereas the two facets are almost $\mathrm{Cu}$-pure (this is the same for the equivalent infinite surfaces). As both facets are coupled with edges, the segregation on edges enhances (in absolute value) the alloying contribution to the permutation enthalpies for the facets. Moreover, we recall that (111) facets are more coupled with edges than (001) facets (in a ratio of 3 to 1 in the present case, see Table II). As a result, the segregation isotherm of the (111) facets is more shifted towards lower $\Delta \mu$ than the (001) facets one, inducing even a reversal in the sequence of the transitions affecting the facets. Actually, as the coupling between edges and facets depends on the size of the nanoparticles, the observed reversal must disappears beyond a critical size. We show Figure 9 the evolution of the critical differences in chemical potentials as a function of the nanoparticle size for the facets and the edges. The sequence of phase transitions for the semi-infinite (100) and (111) surfaces is recovered for nanoparticles of order higher than 16. Moreover, this shows that a reversal exists also between the phase transitions affecting the edges and the (111) facets for nanoparticles of order less than 9. However, this sequence of transitions depends on the crystallographic shape of the considered nanoparticle. In fact, for a truncated octahedron, the coupling between edges and (111) facets is weaker than for a cuboctahedron with the same size, as the $(111) \mathrm{F} /(001) \mathrm{F}$ area ratio is larger. This leads to a shift of the (111)F segregation isotherm towards higher $\Delta \mu$, that may even suppress the reversal in the sequence of phase transitions observed for the cuboctahedron.

Similarly to the high-temperature analysis, we now show in Figure 8 the segregation isotherms in more convenient ways (as a function of $c_{\text {core }}$, Fig. 8a, and as a function of $N_{A g}$, Fig. 8b) to connect with the 'real world' of bimetallic nanoparticles, which is canonical in essence. While first-order phase transitions occur, the connection between the grand canonical and the canonical representations is less straightforward. In fact, the jump in $p$-concentration at $\Delta \mu_{c}^{p}$ from $c_{\text {low }}^{p}$ to $c_{\text {high }}^{p}, c_{\text {low }}^{p}$ (resp. $c_{\text {high }}^{p}$ ) being the solubility limits of the low (resp. high) part of the isotherms, is replaced with a continuous increase of the silver enrichment that corresponds to a mixing of the $\mathrm{Cu}$-rich and Ag-rich phases tuned by the lever rule. As $\Delta \mu$ and $c_{\text {core }}$ are related following Eq. (9), informations contained within the two representations are similar (see Fig. 8a). However, the representation in $c_{\text {core }}$ allows a better view of the segregation behavior as it accounts moreover for the Ag-enrichment factor, 
$c_{p} / c_{\text {core }}$. On the contrary, if the representation in $N_{A g}$ (see Fig. $8 \mathrm{~b}$ ) can describe the silver enrichment of the external shell, it misses all the informations about the possible existence of phase transitions. Actually, since a segregation isotherm is well separated from the others within the $c_{\text {core }}$ representation, e.g., the (001)F isotherm in Fig. 8a, it leads to a continuous and linear isotherm within the $N_{A g}$ representation, independently of the presence of a firstorder phase transition. This shows that both representations are not strictly equivalent. The one using $c_{\text {core }}$ contains more information than the one using $N_{A g}$.

To emphasize this point, we look at the segregation behavior of the nanoparticle external shell with a less detailed level of description, but much closer to what we could expect from experimental measurements (see Fig. 10). Actually, the quantities that should be well determined by experiments are the mean Ag concentration of the entire external shell and the total number of $\mathrm{Ag}$ and $\mathrm{Cu}$ atoms in the nanoparticle, and to some extent, the $\mathrm{Ag}$ core concentration. This is presented in Fig. 10 for the two studied temperatures, both using $c_{\text {core }}$ and $N_{A g}$ representations. We compare also the segregation isotherm obtained by averaging the four segregation isotherms of the different classes of sites of the external shell, i.e., $c_{\text {shell }}=\sum_{p} N_{p} c_{p} / \sum_{p} N_{p}$, to the mean segregation isotherm given by Eq. 5 in which the energetic parameters are obtained by averaging the energetic parameters of the different classes of sites of the external shell, i.e. $\Delta H_{\text {shell }}^{\text {perm }}=\sum_{p} N_{p} \Delta H_{p}^{p e r m} / \sum_{p} N_{p}$. Doing this, it is interesting to note that vertices and edges represents only $20 \%$ of the nanoparticle external shell. The results are as follows:

- At high temperatures, the averaged segregation isotherms curve and the mean segregation isotherm are almost superimposed, both using $c_{\text {core }}$ and $N_{A g}$ representations.

- At low temperatures, while the two isotherms are strictly the same using $N_{A g}$ representation, a difference exists using $c_{\text {core }}$ representation: the averaged isotherms curve exhibits three distinct first-order phase transitions, each one corresponding to one of the transitions that affects the edges and the two facets, whereas the mean isotherm exhibits a unique first-order phase transition, corresponding to a transition affecting the entire external shell.

This shows again that experimental evidences of these phase transitions in nanoparticles will need the knowledge of core concentration of the segregating species and not only its nominal concentration. Actually, it would require to consider alloys with a weaker tendency to phase 
separate in the bulk than $\mathrm{Cu}-\mathrm{Ag}$ to increase the solubility range, e.g., $\mathrm{Cu}-\mathrm{Ni}$. Current works aim at drawing a more extended view of segregation in nanoparticles as a function of the bimetallic nanoparticle energetic parameters $(\tau$ and $\epsilon)$, their size and their shape.

\section{CONCLUSION}

In this work, we compare the segregation behavior of semi-infinite alloy surfaces with nanoparticles for a system that exhibits a strong tendency to phase separation. First, we transfer the mean-field formalism commonly used for surfaces to nanoparticles. Whereas surfaces are described as a stacking of atomic planes, each plane having the same number of atoms, the external shell of nanoparticles is described as an ensemble of classes of sites (vertices, edges and facets), each class owning a different number of atoms.

Studying at high temperature allows us to compare the nanoparticle and its equivalent infinite surfaces. The segregation on the different sites of the nanoparticle is very similar to the segregation of the equivalent infinite surfaces, the enrichment hierarchy being tuned by the number of broken bonds. Moreover, as the slopes of the isotherms at $\Delta \mu_{p}^{c}$ are proportional to the coordination number, this leads to an inversion of the segregation hierarchy between (001) and (111) facets as a function of the bulk concentration (or $\Delta \mu$ ).

At low temperatures, the mean-field formalism predicts a succession of well-distinct firstorder phase transitions for equivalent infinite surfaces with an increasing value of $\Delta \mu_{c}^{p}$ when considering the orientations in the order (110), (001) and (111). For the nanoparticle, there is also a succession of distinct phase transitions for each class of sites but not in the same order, the transition for the (111) facets occurring at lower $\Delta \mu$ than for the (001) facets. This reversal is due to the coupling of facets with edges, that is stronger for the (111) orientation than for the (001) one. Actually, as this coupling depends on the size of the cluster, the observed reversal must disappear for high-order nanoparticles. Moreover, this peculiar phase transition reversal is closely related to the nanoparticle shape. In fact, for a truncated octahedron, the coupling between (111) facets and edges would be weakened (since the $(001) \mathrm{F} /(111) \mathrm{F}$ area ratio is lower than for a cuboctahedron) so that the inversion would probably not take place.

Among the arising prospects from this study, we point out the following ones:

- a first-order phase transition leads to a Van der Waals loop within the semi-grand 
canonical ensemble, corresponding to a mixing of a Cu-rich phase and an Ag-rich phase within the canonical ensemble. At the coexistence point, $\left(\Delta \mu_{p}^{c}, c_{p}=0.5\right)$, both phases have the same free energy. Therefore, whereas the equilibrium within the semigrand canonical ensemble is obtained by a dynamical superimposition of both states during the equilibrium procedure (each one having the same occurrence probability), it is simply obtained by separating the system into two phases within the canonical ensemble. However, when considering nanoparticles, a same segregation isotherm corresponds to several equivalent finite-size systems, e.g., the (001)F segregation isotherm is representative of six (001) facets. Thus, we shall wonder whether the equilibrium is characterized by a phase separation on each facet or by a mixture of $\mathrm{Cu}$-rich and Ag-rich facets.

- Once the external shell is filled up with Ag, how does this Ag superficial enrichment propagate towards the nanoparticle core? Relatively to semi-infinite surfaces and to thin film geometries, the finite-size of nanoparticles modifies possibly the wetting behavior that may occur $[29,44]$.

Finally, we show that the comparison of the Ising model with experiments may not be straightforward due to various complicating factors in experiments: (i) the knowledge of the core concentration of the segregating species is essential to bring prominence to phase transitions and it may not be reachable by experimental techniques. (ii) For real systems, the alloying pair interactions may differ from one site to another depending on the site location within the nanoparticle (external shell, core, etc...) [29]. (iii) For systems with strong size effect, i.e., with constituents that exhibit a large difference in their atomic radii, the Isingtype phase transitions are often accompanied by structural phase transitions [3]. Obviously, the present model cannot account for such a phenomenon, even if atomic relaxation effects can be partially incorporated in the energetic parameters of the Ising model $[3,29,45,46]$. (iv) Last but not least, if the critical temperature is low, very slow kinetics may prevent the experimental observation of the transition.

Thus, the present work can be considered as a first step for the understanding of the segregation behavior of bi-metallic nanoparticles and we do hope that it will be a useful guide for further studies and their interpretation.

Acknowledgements. It is a great pleasure to thank C. Mottet, G. Tréglia, I. Braems, R. 
Tétot, and V. Moreno for very fruitful discussions. We are also indebted to one the referees for having noticed us of the possible difference in the sequence of phase transitions between a cuboctahedron and a truncated octahedron.

[1] Y. Gauthier and R. Baudoing, in Surface Segregation and Related Phenomena (P. A. Dowben and A. Miller (Eds.), CRC, Boca Raton, 1990).

[2] G. Tréglia, B. Legrand, F. Ducastelle, A. Saúl, C. Gallis, I. Meunier, C. Mottet, and A. Senhaji, Comput. Mat. Sci. 15, 196 (1999).

[3] J. Creuze, F. Berthier, R. Tétot, and B. Legrand, Surf. Sci. 491, 1 (2001); J. Creuze, F. Berthier, R. Tétot, and B. Legrand, ibid., 491, L651 (2001).

[4] J. Creuze, Def. Diff. Forum 203-205, 3 (2002).

[5] J. H. Sinfelt, in Bimetallic Catalysts: Discoveries, Concepts and Applications (Wiley, New York, 1983).

[6] D. F. Ollis, J. Catal. 23, 131 (1971).

[7] J. J. Burton, E. Hyman, and D. G. Fedak, J. Catal. 37, 106 (1975).

[8] N.-H. Tsai, F. F. Abraham, and G. M. Pound, Surf. Sci. 77, 465 (1978).

[9] J. K. Strohl and T. S. King, J. Catal. 116, 540 (1989); J. K. Strohl and T. S. King, ibid. 118, 53 (1989).

[10] J. M. Montejano-Carrizales and J. L. Morán-López, Surf. Sci. 239, 169 (1990); J. M. Montejano-Carrizales and J. L. Morán-López, ibid. 239, 178 (1990).

[11] A. M. Schoeb, T. J. Raeker, and A. E. De Pristo, Surf. Sci. 278, L125 (1992).

[12] L. Yang, T. J. Raeker, and A. E. De Pristo, Surf. Sci. 290, 195 (1993).

[13] O. L. J. Gijzeman, Appl. Surf. Sci. 64, 9 (1993).

[14] L. Yang and A. E. De Pristo, J. Catal. 148, 575 (1994).

[15] Ling Zhu and A. E. De Pristo, J. Chem. Phys. 102, 5342 (1995).

[16] Ling Zhu and A. E. De Pristo, J. Catal. 167, 400 (1997); Ling Zhu et al., ibid. 167, 412 (1997).

[17] J. L. Rousset, A. J. Renouprez, and A. M. Cadrot, Phys. Rev. B 58, 2150 (1998).

[18] S. Darby, T. V. Mortimer-Jones, R. L. Johnston, and C. Roberts, J. Chem. Phys. 116, 1536 (1995).

[19] C. Mottet, G. Tréglia, and B. Legrand, Phys. Rev. B 66, 045413 (2002). 
[20] F. Baletto, C. Mottet, A. Rapallo, G. Rossi, and R. Ferrando, Surf. Sci. 566-568, 192 (2004).

[21] L. Rubinovich and M. Polak, Phys. Rev. B 69, 155405 (2004); M. Polak and L. Rubinovich, Int. J. Nanosci. 3, 625 (2004); M. Polak and L. Rubinovich, Phys. Rev. B 125426 (2005).

[22] G. Wang, M. A. Van Hove, P. N. Ross, and M. I. Baskes, J. Chem. Phys. 121, 5410 (2004).

[23] G. Wang, M. A. Van Hove, P. N. Ross, and M. I. Baskes, J. Chem. Phys. 122, 024706 (2005).

[24] C. R. Helms, Surf. Sci. 69, 689 (1977).

[25] A. Saúl, B. Legrand, and G. Tréglia, Phys. Rev. B 50, 1912 (1994).

[26] Y. Teraoka and T. Seto, Surf. Sci. 255, 209 (1991).

[27] Y. Liu and P. Wynblatt, Surf. Sci. 241, L21 (1991).

[28] J. Eugène, B. Aufray, and F. Cabané, Surf. Sci. 241, 1 (1991).

[29] F. Berthier, J. Creuze, R. Tétot, and B. Legrand, Phys. Rev. B 65, 195413 (2002).

[30] K. Binder, in The Monte Carlo Method in Condensed Matter Physics (Springer Verlag, Berlin, 1995).

[31] J. L. Morán-López and L. M. Falicov, Phys. Rev. B 18, 2549 (1978).

[32] F. Baletto and R. Ferrando, Rev. Mod. Phys. 77, 371 (2005).

[33] F. Baletto, R. Ferrando, A. Fortunelli, F. Montalenti, and C. Mottet, J. Chem. Phys. 116, $3856(2002)$.

[34] C. Mottet, J. Goniakowski, F. Baletto, R. Ferrando, and G. Tréglia, Phase Transitions 77, 101 (2004).

[35] F. Ducastelle, in Order and Phase Stability in Alloys (North-Holland, Amsterdam, 1991).

[36] W. L. Bragg and J. L. Williams, Proc. Roy. Soc. A 145, 699 (1934).

[37] R. H. Fowler and E. H. Guggenheim, in Statistical Thermodynamics (Cambridge University Press, Cambridge, 1960).

[38] V. S. Sundaram and P. Wynblatt, Surf. Sci. 52, 569 (1975).

[39] M. A. Hoffmann and P. Wynblatt, J. Vac. Sci. Techon. A 9, 27 (1991).

[40] J. Eugène, B. Aufray, and F. Cabané, Surf. Sci. 273, 372 (1992).

[41] Y. Liu and P. Wynblatt, Surf. Sci. 290, 335 (1993).

[42] Y. Liu and P. Wynblatt, J. Vac. Sci. Technol. A 12, 255 (1994).

[43] M. E. Fisher, Rep. Prog. Phys. 30, 615 (1967).

[44] K. Binder, P. Niebala, and V. Pereyra, Z. Phys. B 104, 81 (1997).

[45] B. Legrand, A. Saúl, and G. Tréglia, Mat. Sci. Forum 155-156, 165 (1994). 
[46] F. Berthier, B. Legrand, and G. Tréglia, Acta Mater. 47, 2705 (1999). 
VI. TABLES

\begin{tabular}{|c|c|c|c|c|c||c|}
\hline \hline & $\mathrm{V}$ & $\mathrm{E}$ & $(001) \mathrm{F}$ & $(111) \mathrm{F}$ & core & $N_{p}$ \\
\hline $\mathrm{V}$ & 0 & 48 & 0 & 0 & 12 & 12 \\
& $(0)$ & $(48)$ & $(0)$ & $(0)$ & $(12)$ & $(12)$ \\
\hline $\mathrm{E}$ & 48 & $48(n-1)$ & $24(n-1)$ & $48(n-2)$ & $48(n-1)$ & $24(n-1)$ \\
& $(48)$ & $(432)$ & $(216)$ & $(384)$ & $(432)$ & $(216)$ \\
\hline$(001) \mathrm{F}$ & 0 & $24(n-1)$ & $24(n-1)(n-2)$ & 0 & $24(n-1)^{2}$ & $6(n-1)^{2}$ \\
& $(0)$ & $(216)$ & $(1728)$ & $(0)$ & $(1944)$ & $(486)$ \\
\hline$(111) \mathrm{F}$ & 0 & $48(n-2)$ & 0 & $24(n-2)(n-3)$ & $12(n-1)(n-2)$ & $4(n-1)(n-2)$ \\
& $(0)$ & $(384)$ & $(0)$ & $(1344)$ & $(864)$ & $(288)$ \\
\hline \hline
\end{tabular}

Table I: Number of bonds between the different classes of sites, $N_{p, q}$, and total number of $p$-sites, $N_{p}$, as a function of the order of the nanoparticle, $n$. The case $n=10$ is indicated in parentheses.

\begin{tabular}{|c|c|c|c|c|c||c|c|c|c|}
\hline \hline & $\mathrm{V}$ & $\mathrm{E}$ & $(001) \mathrm{F}$ & $(111) \mathrm{F}$ & core & & $q=0$ & $q=1$ & $q=2$ \\
\hline $\mathrm{V}$ & $0(0)$ & $4(4)$ & $0(0)$ & $0(0)$ & $1(1)$ & $(110)$ ads $p=0$ & 0 & 4 & 1 \\
\hline $\mathrm{E}$ & $0.2(0)$ & $2(2)$ & $1(1)$ & $1.8(2)$ & $2(2)$ & $(110) \mathrm{S} p=0$ & 2 & 4 & 1 \\
\hline$(001) \mathrm{F}$ & $0(0)$ & $0.4(0)$ & $3.6(4)$ & $0(0)$ & $4(4)$ & $(001) \mathrm{S} p=0$ & 4 & 4 & 0 \\
\hline$(111) \mathrm{F}$ & $0(0)$ & $1.3(0)$ & $0(0)$ & $4.7(6)$ & $3(3)$ & $(111) \mathrm{S} p=0$ & 6 & 3 & 0 \\
\hline \hline
\end{tabular}

Table II: coordination numbers, $Z_{p, q}$, (left) for the nanoparticle of order $n=10$, the limit $n \rightarrow \infty\left(Z_{p, q}^{\infty}=\lim _{n \rightarrow \infty} \frac{N_{p, q}}{N_{p}}\right)$ being indicated in parentheses, and (right) for the equivalent infinite surfaces. For the latter, $p$ and $q$ represent the indices of planes parallel to the surface plane $(p=0)$.

\begin{tabular}{|c|c|c|c|c|c|}
\hline & $\mathrm{V}$ & $\mathrm{E}$ & $(001) \mathrm{F}$ & $(111) \mathrm{F}$ & core \\
\hline \hline$\Delta H_{p}^{\text {perm,imp }}$ & 0.380 & 0.532 & 0.608 & 0.684 & 0.912 \\
\hline$\Delta H_{p}^{\text {seg,imp }}$ & -0.532 & -0.380 & -0.304 & -0.228 & 0 \\
\hline$T_{c}^{p}$ & $0(0)$ & $348(348)$ & $619(696)$ & $812(1044)$ & 2088 \\
\hline
\end{tabular}

Table III: Permutation energies in the infinitely dilute limit (in $\mathrm{eV} / \mathrm{at}$ ), $\Delta H_{p}^{\text {perm,imp }}$, segregation energies in the infinitely dilute limit (in $\mathrm{eV} / \mathrm{at}$ ), $\Delta H_{p}^{\text {seg,imp }}$ and mean-field critical temperature of first-order phase transition (in $\mathrm{K}$ ) for a cuboctahedron of order $n=10$, 
$T_{c}^{p}$. For the latter, the values obtained for the equivalent infinite surfaces are indicated in parentheses. 


\section{FIGURE CAPTIONS}

Figure 1: (color online) 3871 atoms cuboctahedron (order $n=10)$. Vertices, edges, $(001)$ facets and (111) facets are colored in black, red, green (square facets) and blue (triangular facets) respectively. This color code will be used for all other figures.

Figure 2: (color online) Segregation isotherms for the different classes of sites of the external shell (a) as function of $\Delta \mu(\mathrm{in} \mathrm{eV})$ and (b) as function of the total number of silver atoms, $N_{A g}$, obtained within a mean-field approximation (lines) and by Monte Carlo simulations (symbols) at $T=1100 \mathrm{~K}$. Vertices (solid line/squares), edges (dashed line/circles), (001) facets (dotted line/up triangles), (111) facets (dashed-dotted line/down triangles), and core (dashed-dotted-dotted line/diamonds) for the representation (b) only.

Figure 3: (color online) Segregation isotherms as function of $\Delta \mu$ (in eV), obtained within the mean-field formalism at $T=1100 \mathrm{~K}$ for the different classes of sites of the external shell of the nanoparticle (lines) and for the equivalent infinite surfaces (symbols). (a) (001) facet (dotted line) and (001) surface (up triangles), (b) (111) facet (dashed-dotted line) and (111) surface (down triangles), (c) vertice (solid line) and adatom on a (110) surface (squares), and (d) edges (dashed line) and (110) surface (circles).

Figure 4: (color online) Superimposition of the segregation isotherms for the four equivalent infinite surfaces as function of $\Delta \mu$ (in eV), obtained within the mean-field formalism at $T=1100 \mathrm{~K}$. Adatom on a (110) surface (solid line), (110) surface (dashed line), (001) surface (dotted line), and (111) surface (dashed-dotted line).

Figure 5: (color online) Superimposition of the segregation isotherms for the four infinite surfaces as function of $\Delta \mu$ (in $\mathrm{eV}$ ), obtained within the mean-field formalism at $T=300$ K. (a) All the thermodynamic states are represented (stable, metastable and unstable). (b) Only stable states are represented. Adatom on a (110) surface (solid line), (110) surface (dashed line), (001) surface (dotted line), and (111) surface (dashed-dotted line).

Figure 6: (color online) Segregation isotherms for the different classes of sites of the external shell of the nanoparticle as function of $\Delta \mu$ (in $\mathrm{eV}$ ), obtained within the mean-field formalism at $T=300 \mathrm{~K}$. All the thermodynamic states are represented. Vertices (solid line), edges (dashed line), (001) facets (dotted line) and (111) facets (dashed-dotted line).

Figure 7: Ground states of the Ising Hamiltonian obtained following Eqs. (17a-17c) (see 
text for more precisions).

Figure 8: (color online) Segregation isotherms for the different classes of sites of the external shell (a) as function of $\Delta \mu$ (in $\mathrm{eV}$ ) or the Ag core concentration, $c_{\text {core }}$, and (b) as function of the total number of silver atoms, $N_{A g}$, obtained within the mean-field formalism at $T=300 \mathrm{~K}$. Only stable states are represented. Vertices (solid line), edges (dashed line), (001) facets (dotted line) and (111) facets (dashed-dotted line), and core (dashed-dotteddotted line) for the representation (b) only.

Figure 9: (color online) Critical differences in chemical potentials of phase transitions, $\Delta \mu_{c}^{p}=\Delta \mu\left(c_{p}=0.5\right)$, as function of the nanoparticle size $n$. Edges (dashed line), (001) facets (dotted line) and (111) facets (dashed-dotted line). The corresponding semi-inifine values are indicated by the straight horizontal lines.

Figure 10: Segregation isotherm averaged over the four classes of sites of the external shell of the nanoparticle (solid lines) and mean segregation isotherm of the external shell of the nanoparticle (dashed lines) (a) as a function of the Ag core concentration, $c_{\text {core }}$, and (b) as a function of the total number of silver atoms, $N_{A g}$, obtained within the mean-field formalism at $300 \mathrm{~K}$ (black) and $1100 \mathrm{~K}$ (grey). Only stable states are represented. 


\section{TABLE CAPTIONS}

Table I: Number of bonds between the different classes of sites, $N_{p, q}$, and total number of $p$-sites, $N_{p}$, as a function of the order of the nanoparticle, $n$. The case $n=10$ is indicated in parentheses.

Table II: Coordination numbers, $Z_{p, q}$, (left) for the nanoparticle of order $n=10$, the limit $n \rightarrow \infty\left(Z_{p, q}^{\infty}=\lim _{n \rightarrow \infty} \frac{N_{p, q}}{N_{p}}\right)$ being indicated in parentheses, and (right) for the equivalent infinite surfaces. For the latter, $p$ and $q$ represent the indices of planes parallel to the surface plane $(p=0)$.

Table III: Permutation energies in the infinitely dilute limit (in $\mathrm{eV} / \mathrm{at}$ ), $\Delta H_{p}^{\text {perm,imp }}$, segregation energies in the infinitely dilute limit (in $\mathrm{eV} / \mathrm{at}$ ), $\Delta H_{p}^{\text {seg,imp }}$ and mean-field critical temperature of first-order phase transition (in $\mathrm{K}$ ) for a cuboctahedron of order $n=10$, $T_{c}^{p}$. For the latter, the values obtained for the equivalent infinite surfaces are indicated in parentheses. 


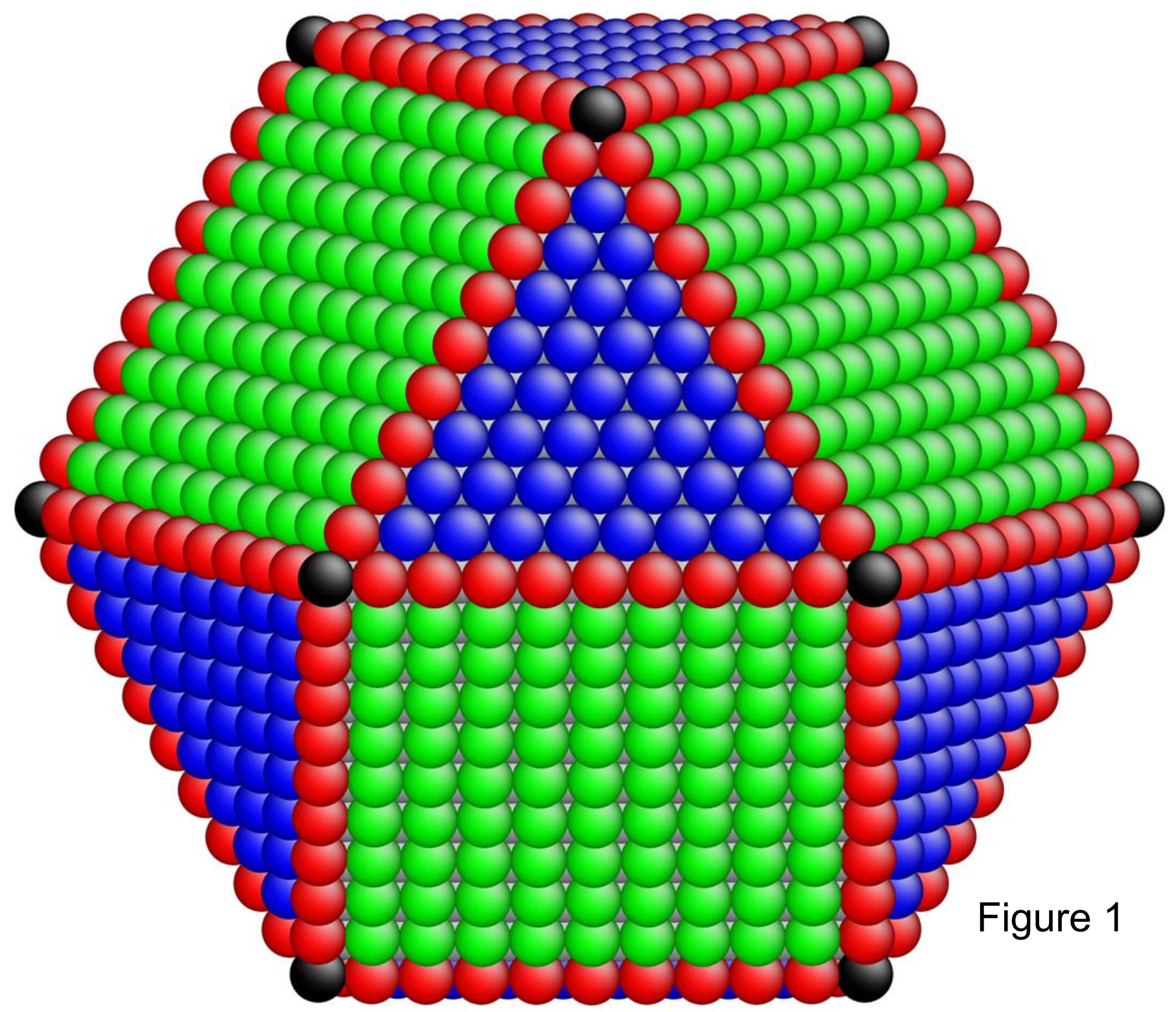




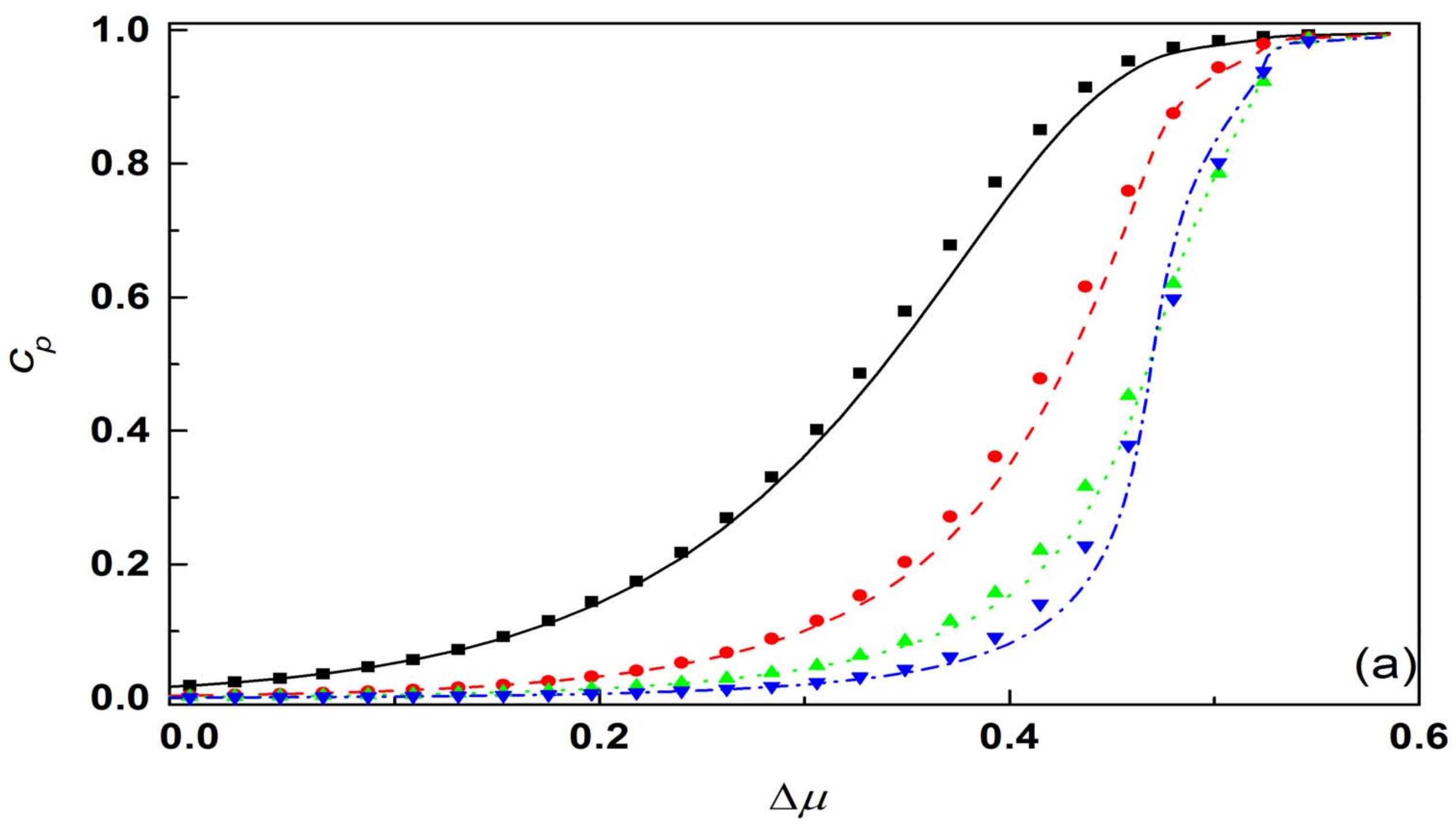




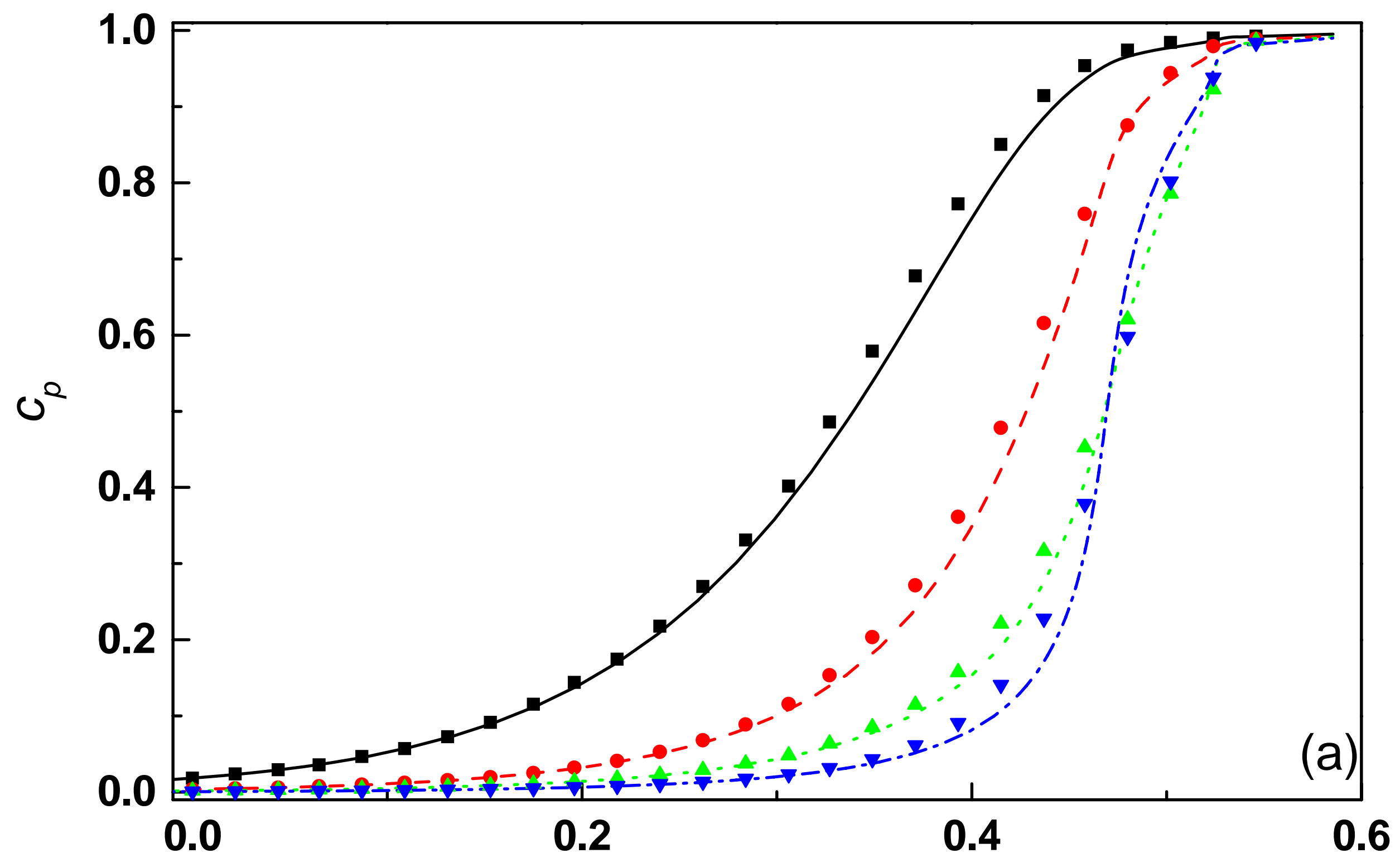

$\Delta \mu$

Figure $2 a$ 


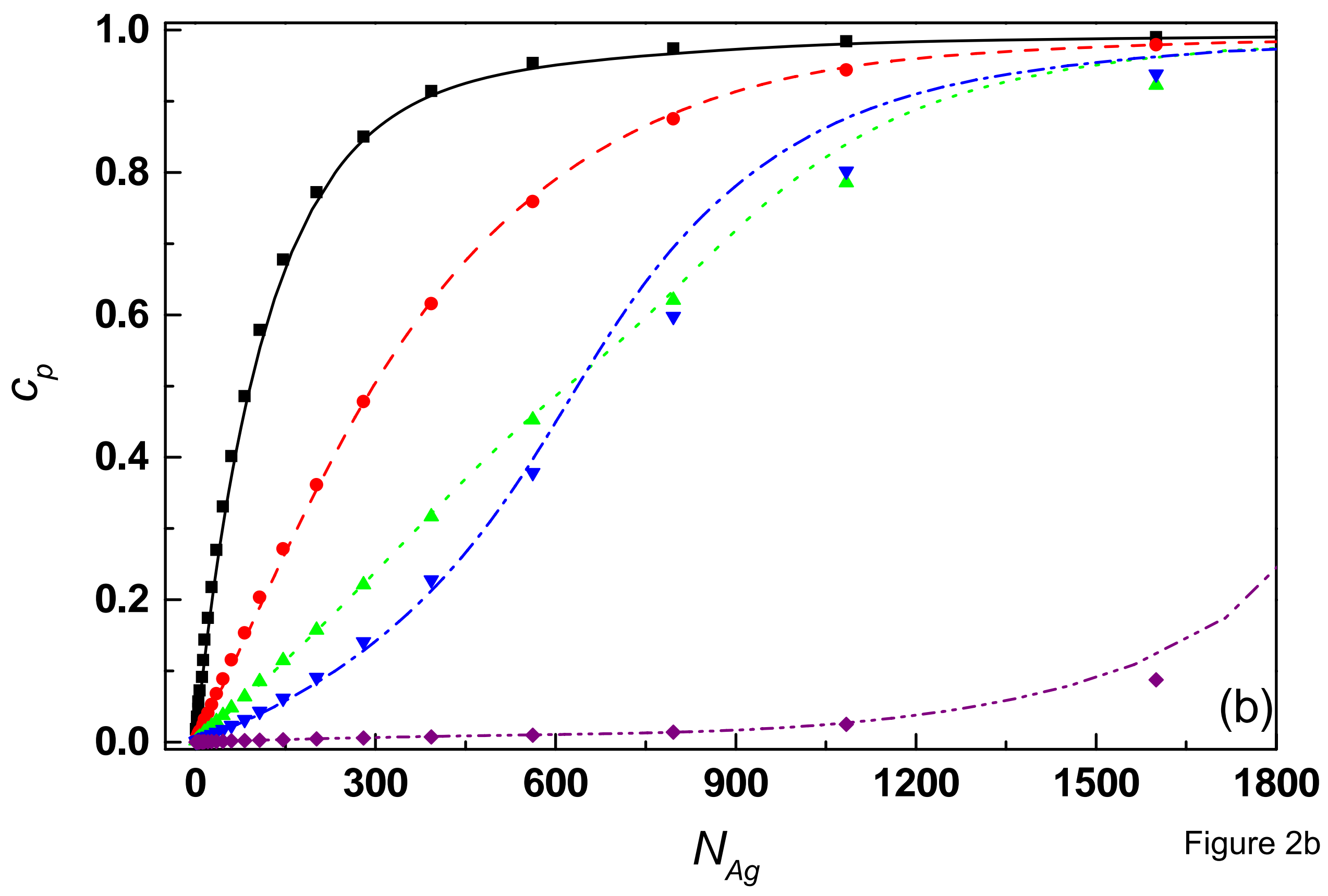




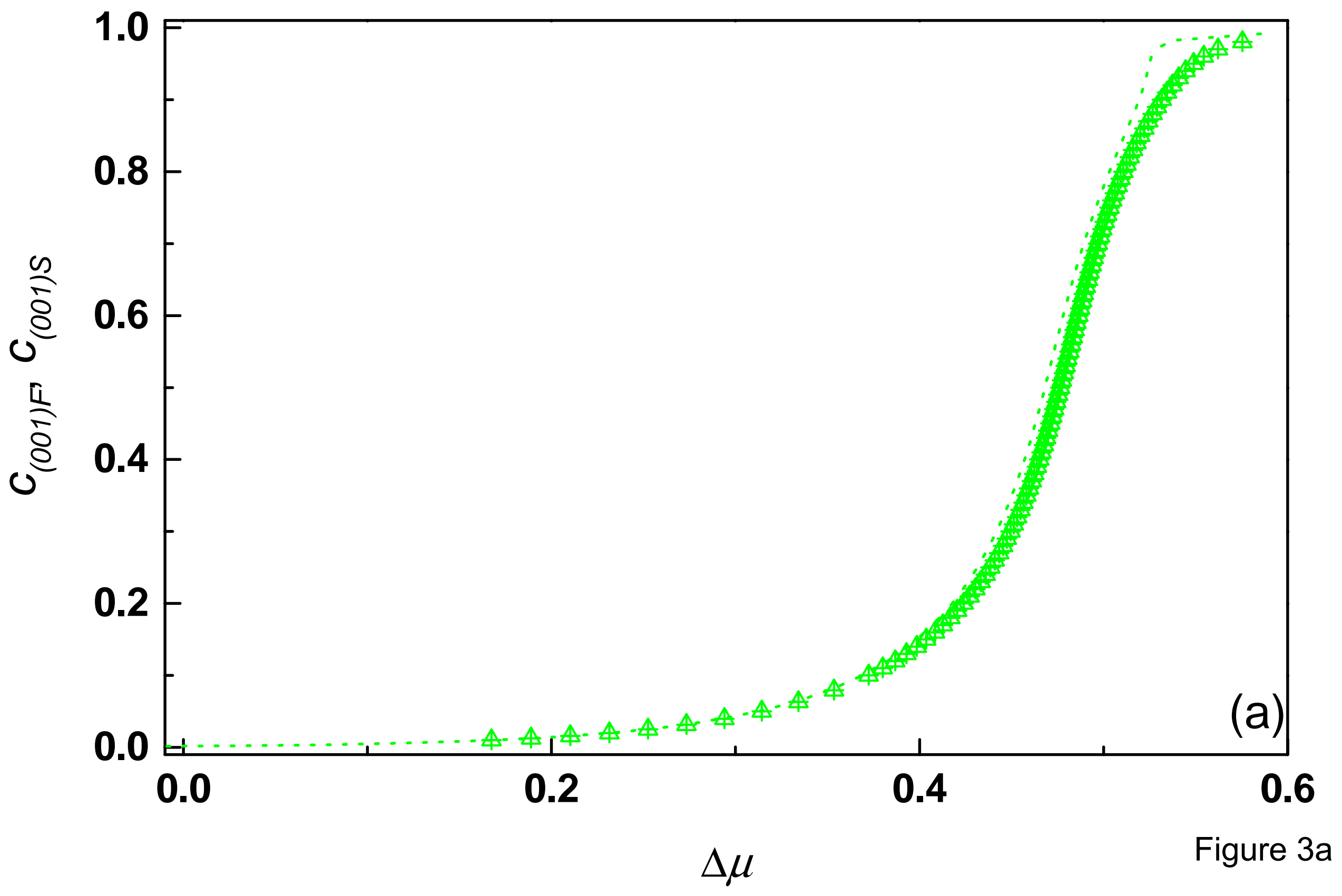




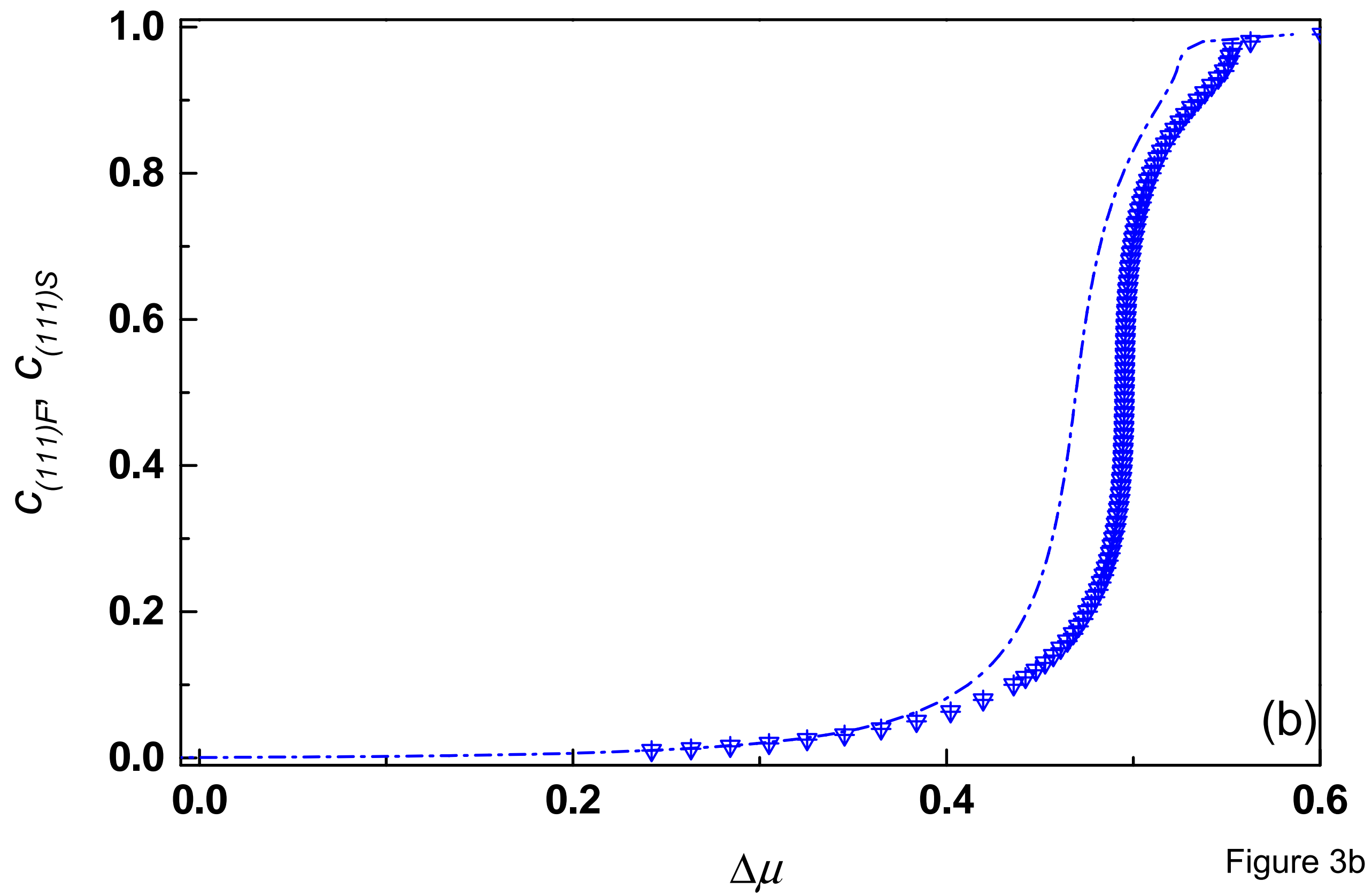




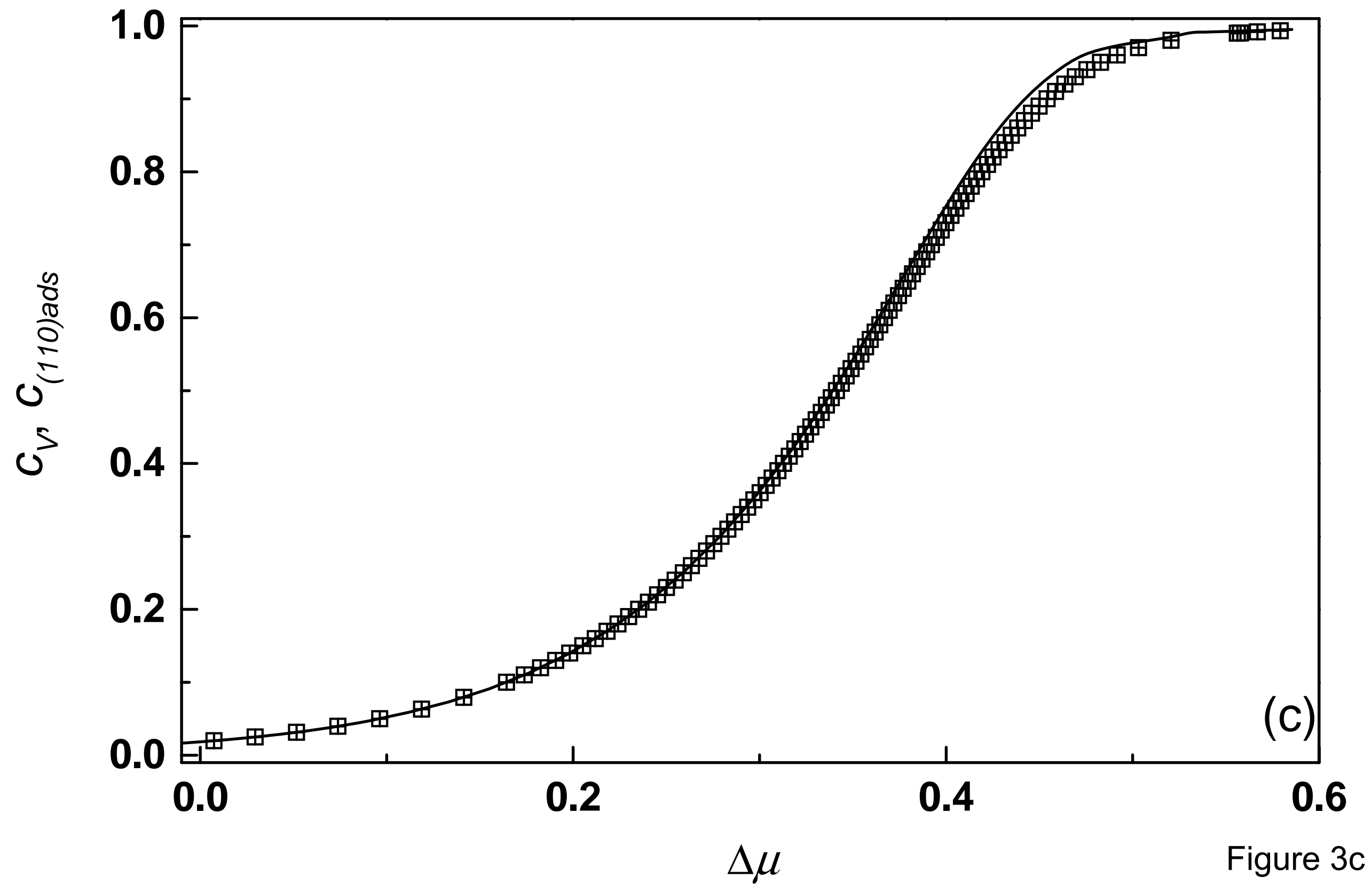




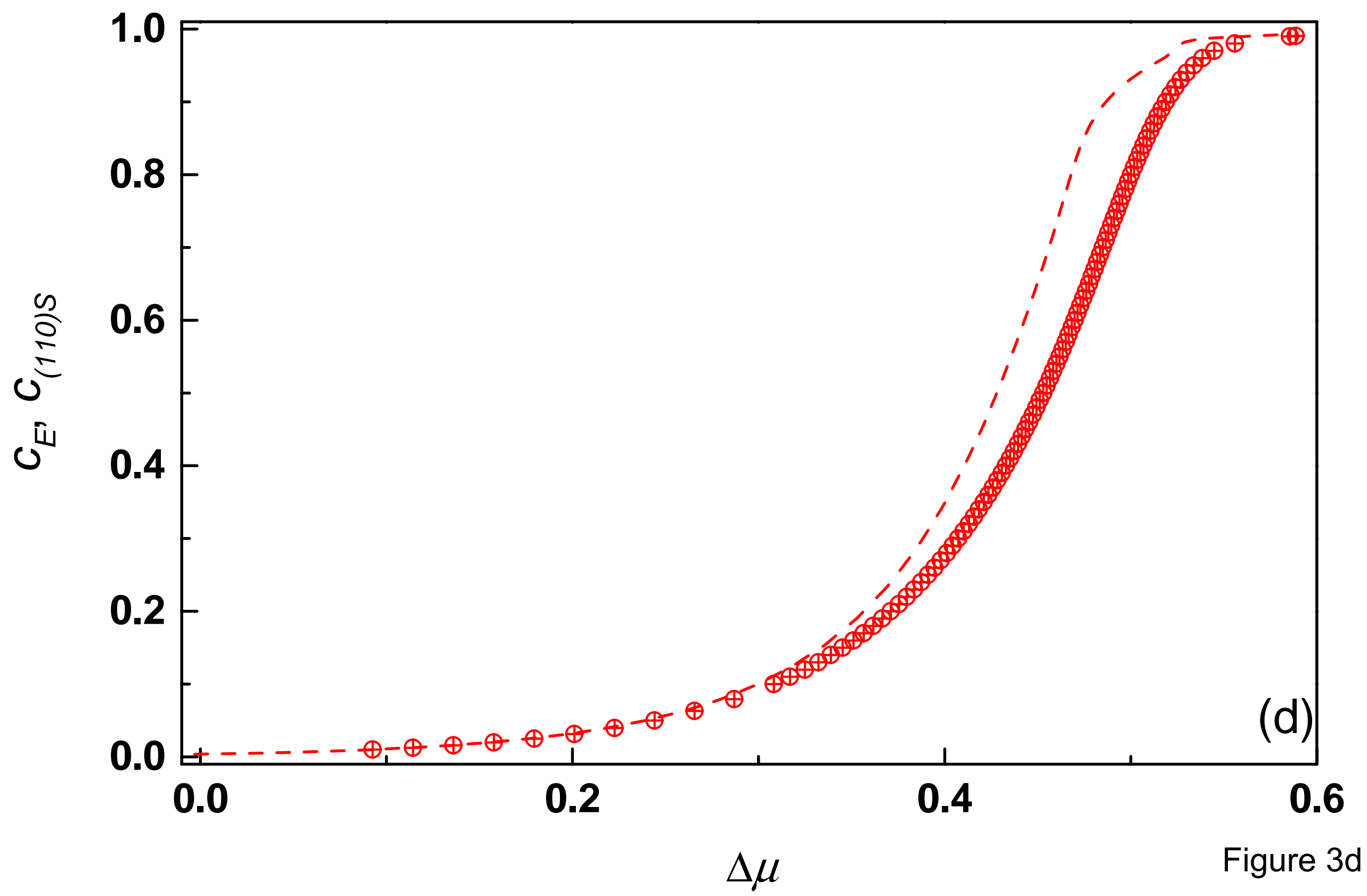




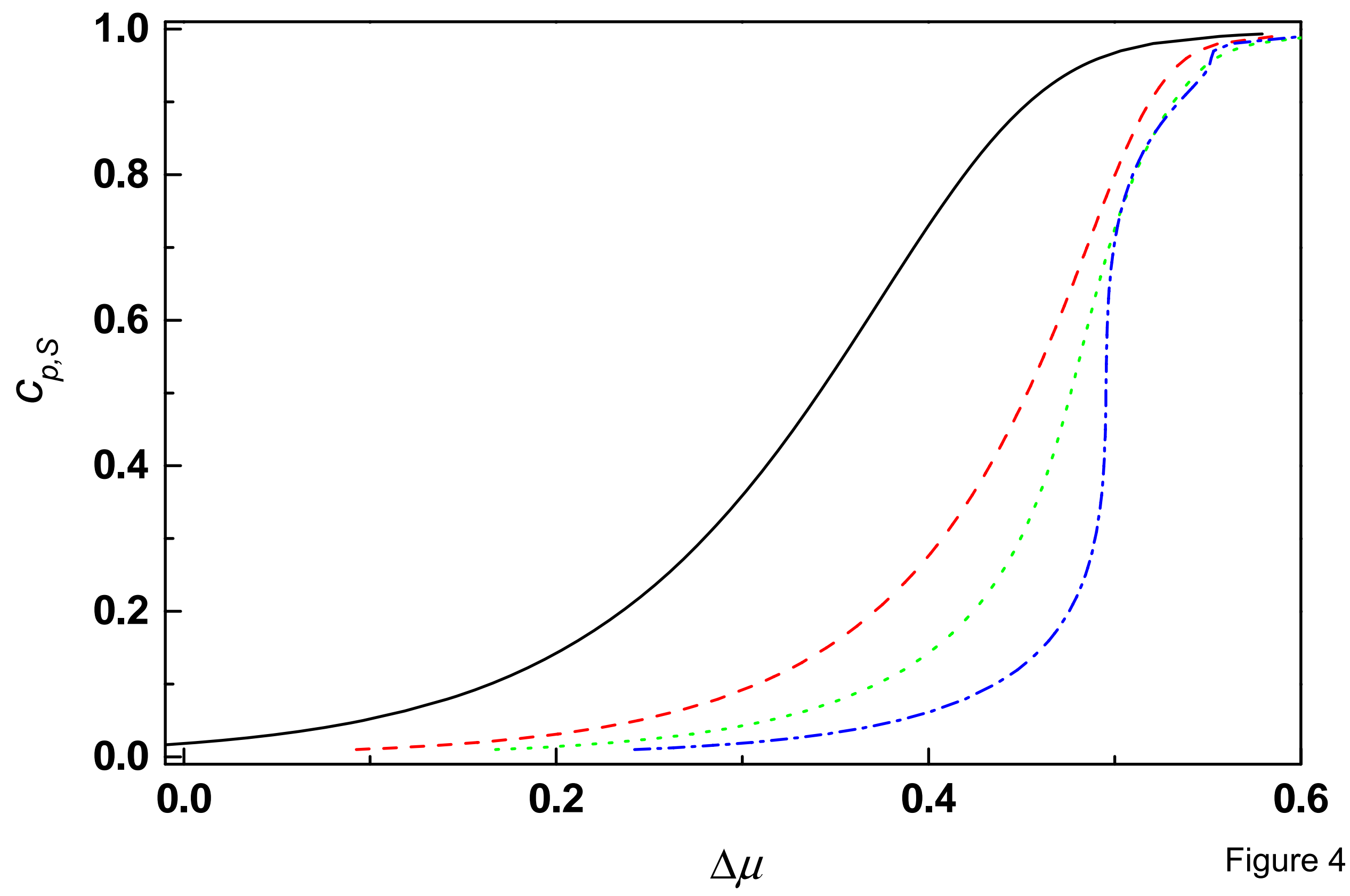




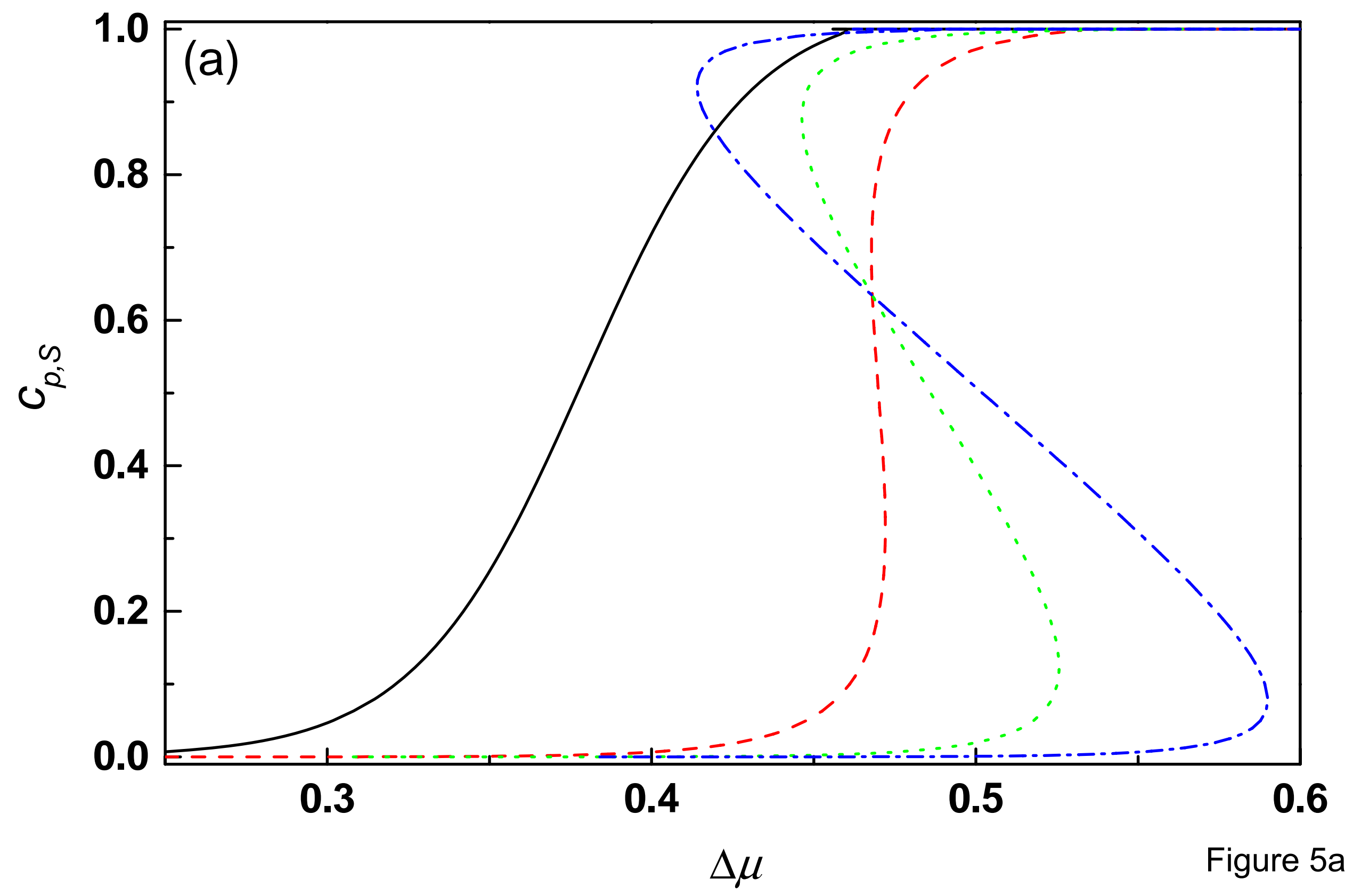




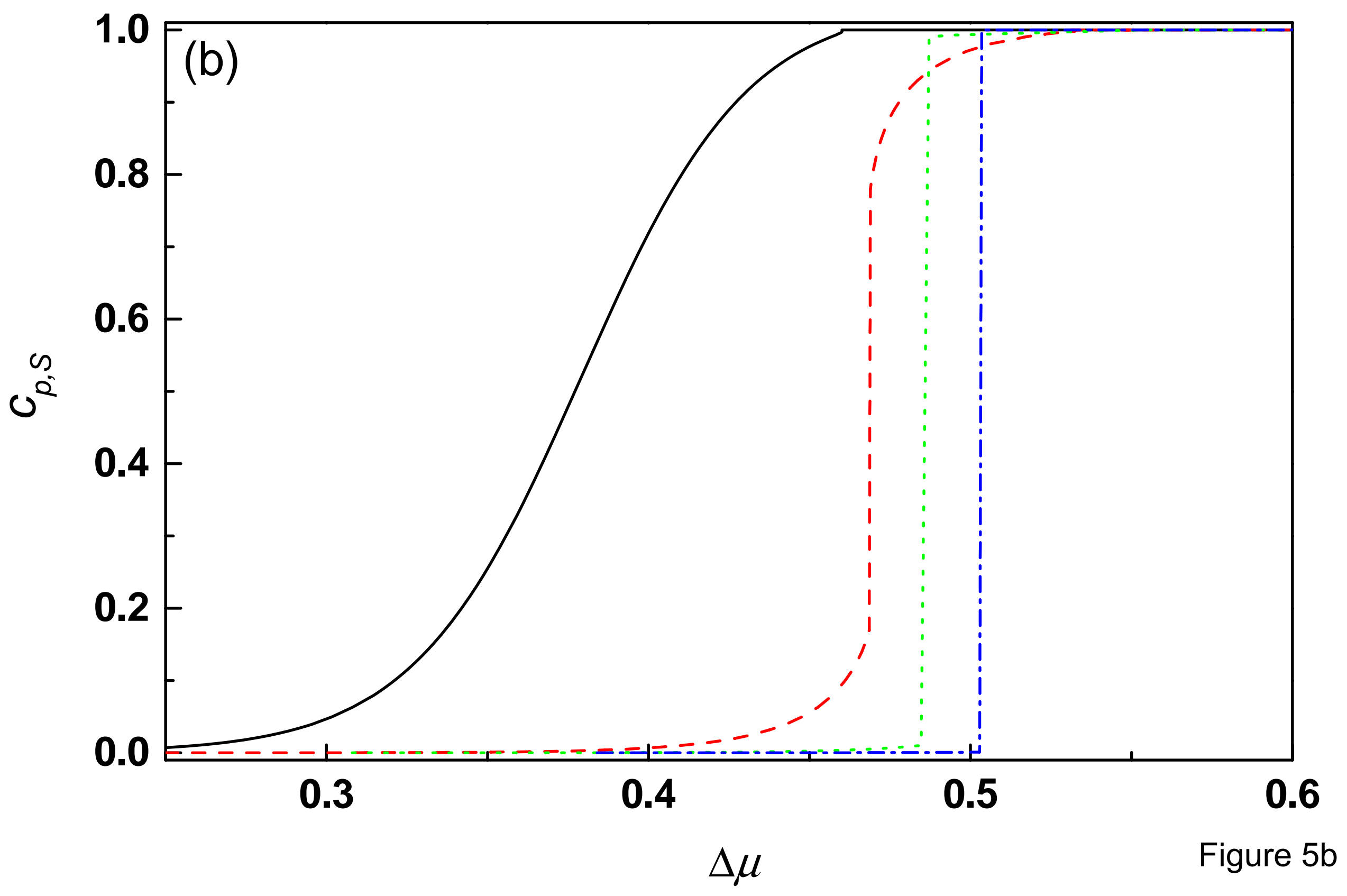




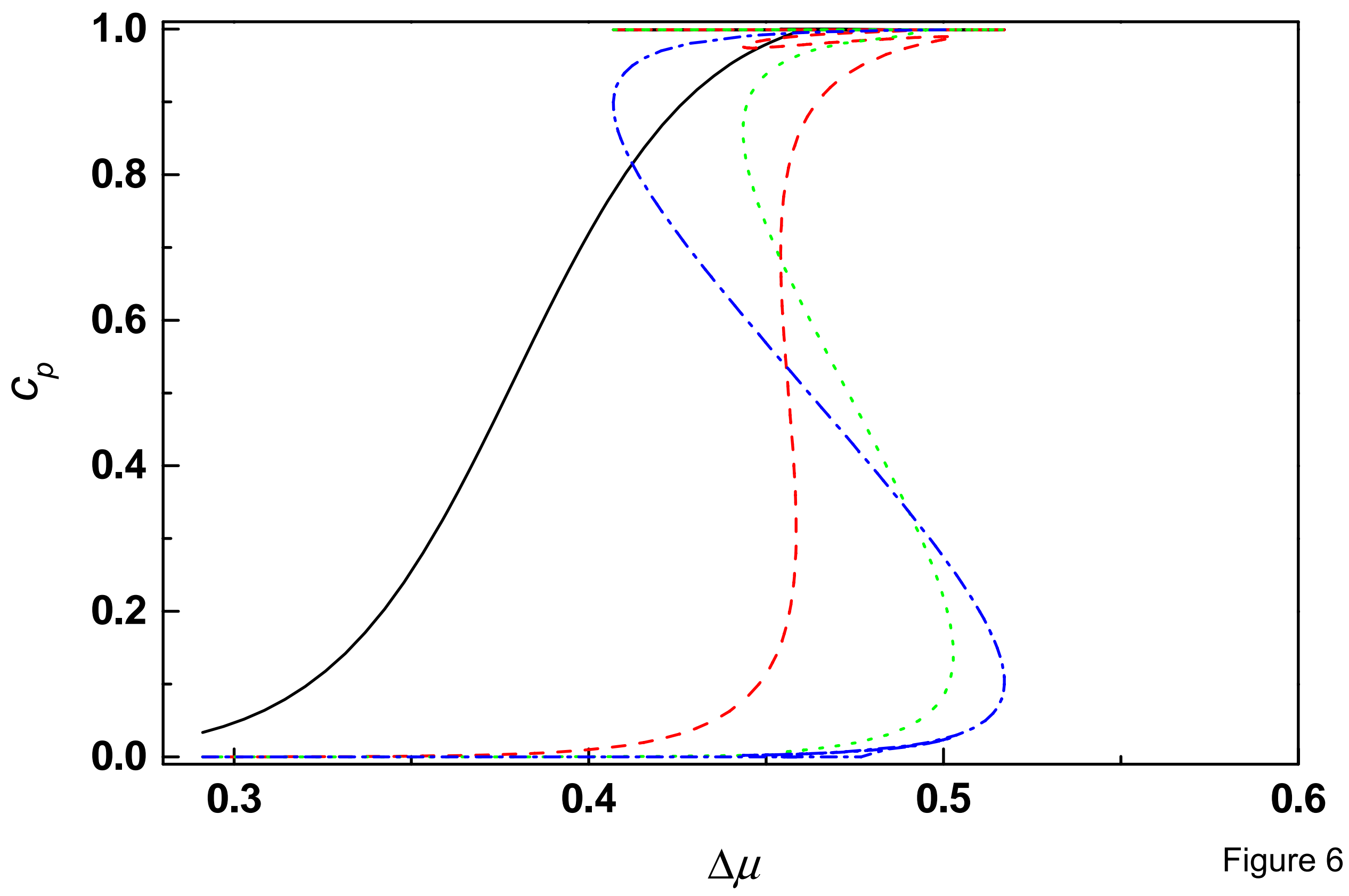




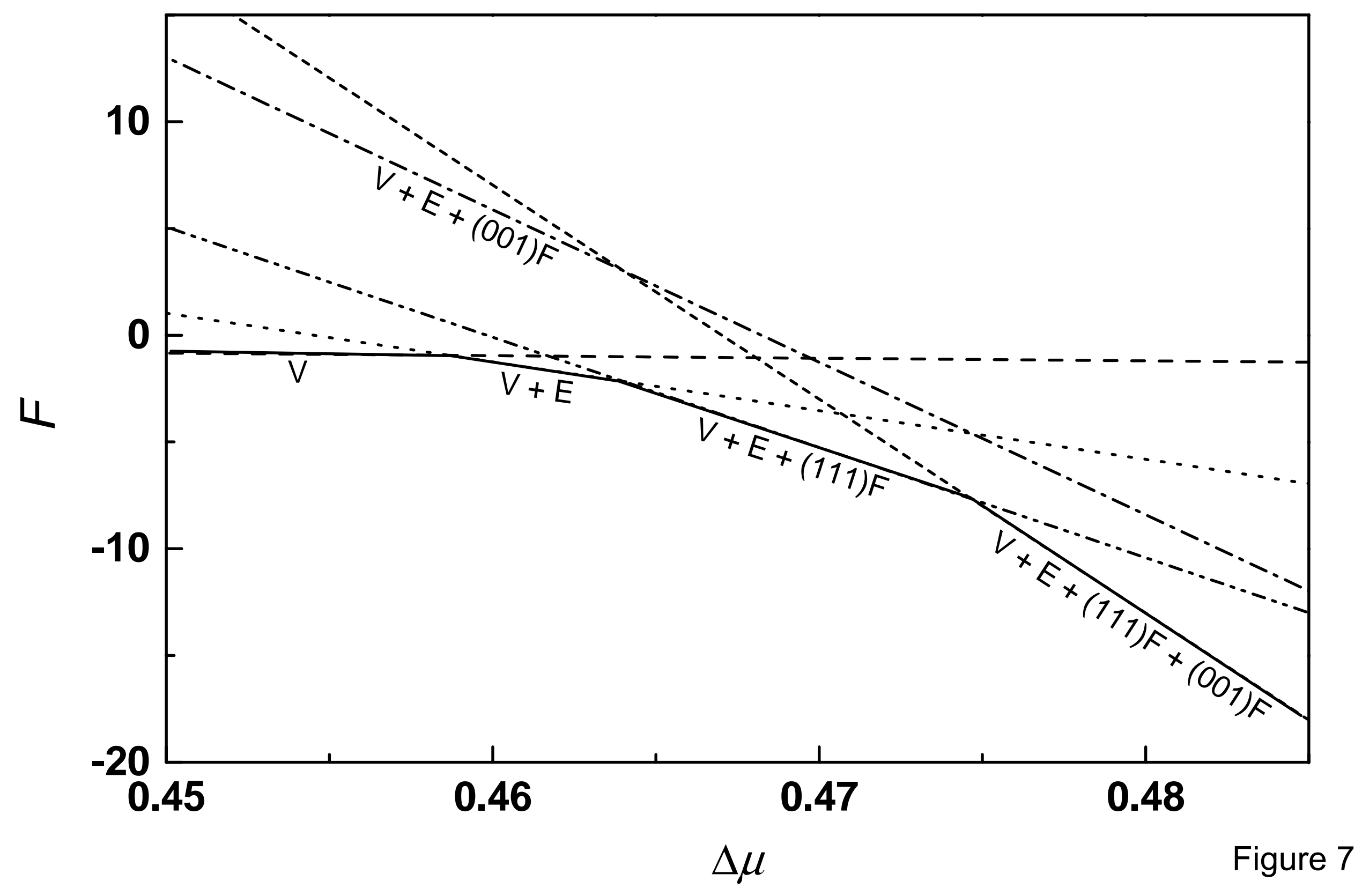




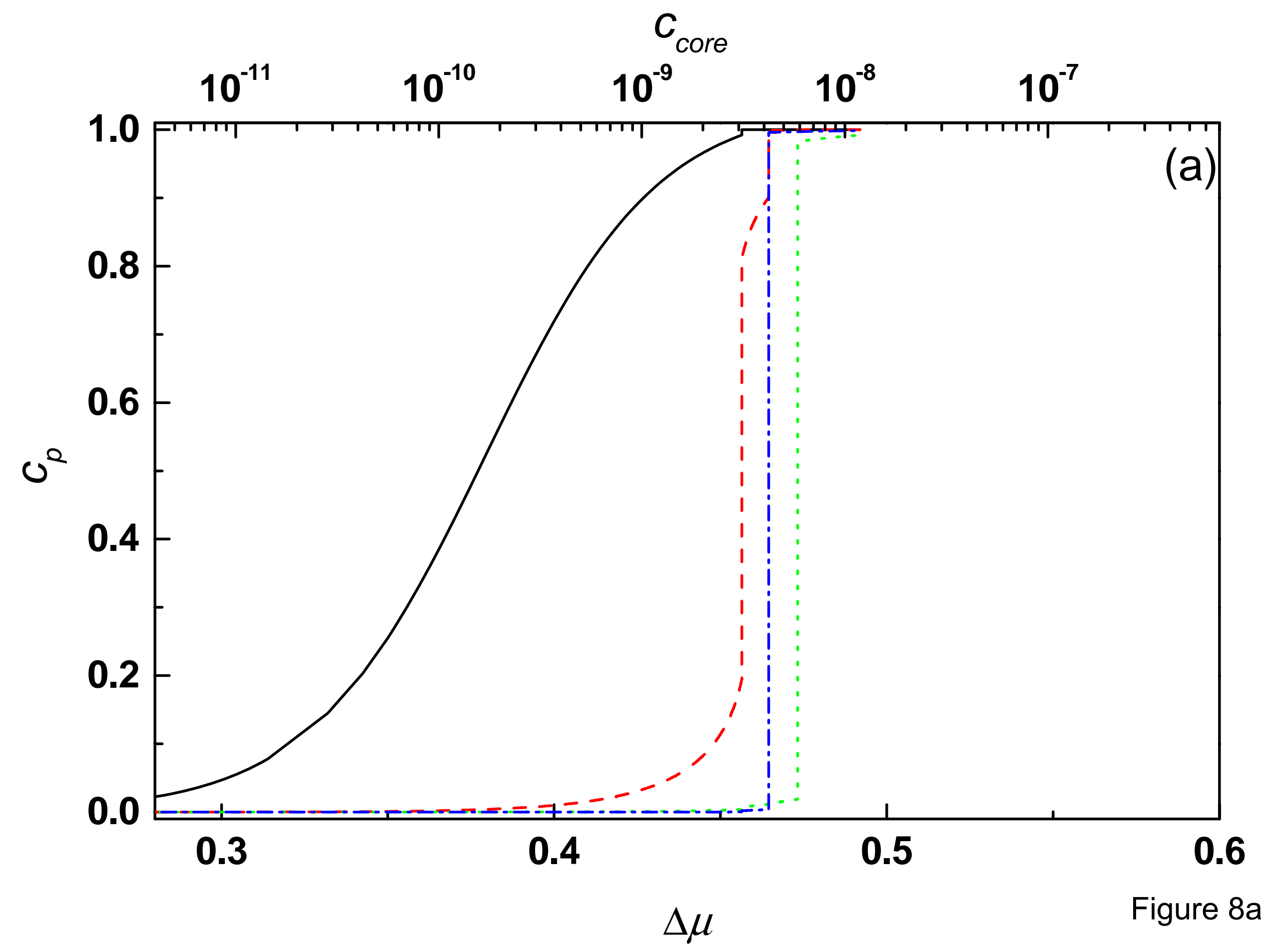




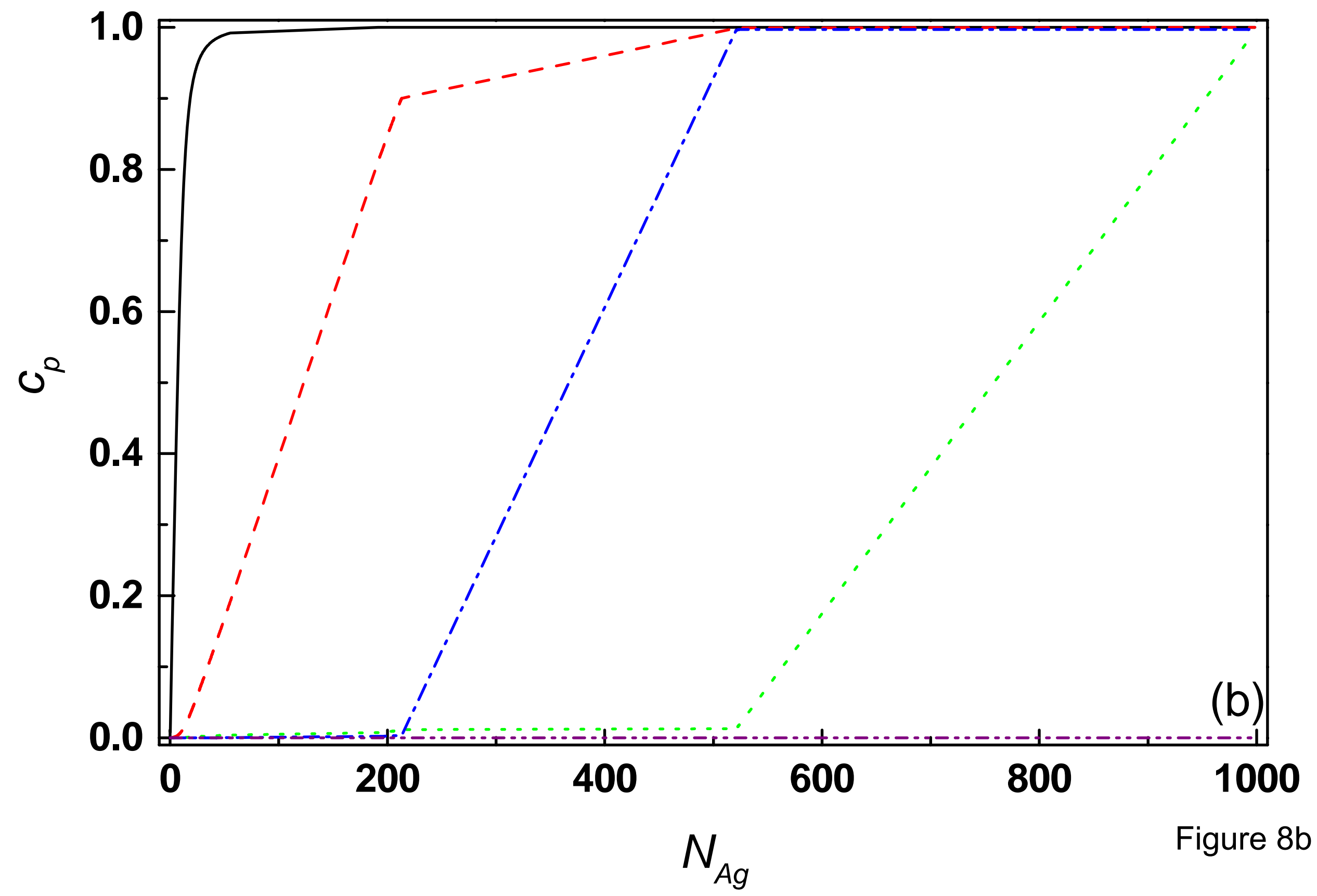




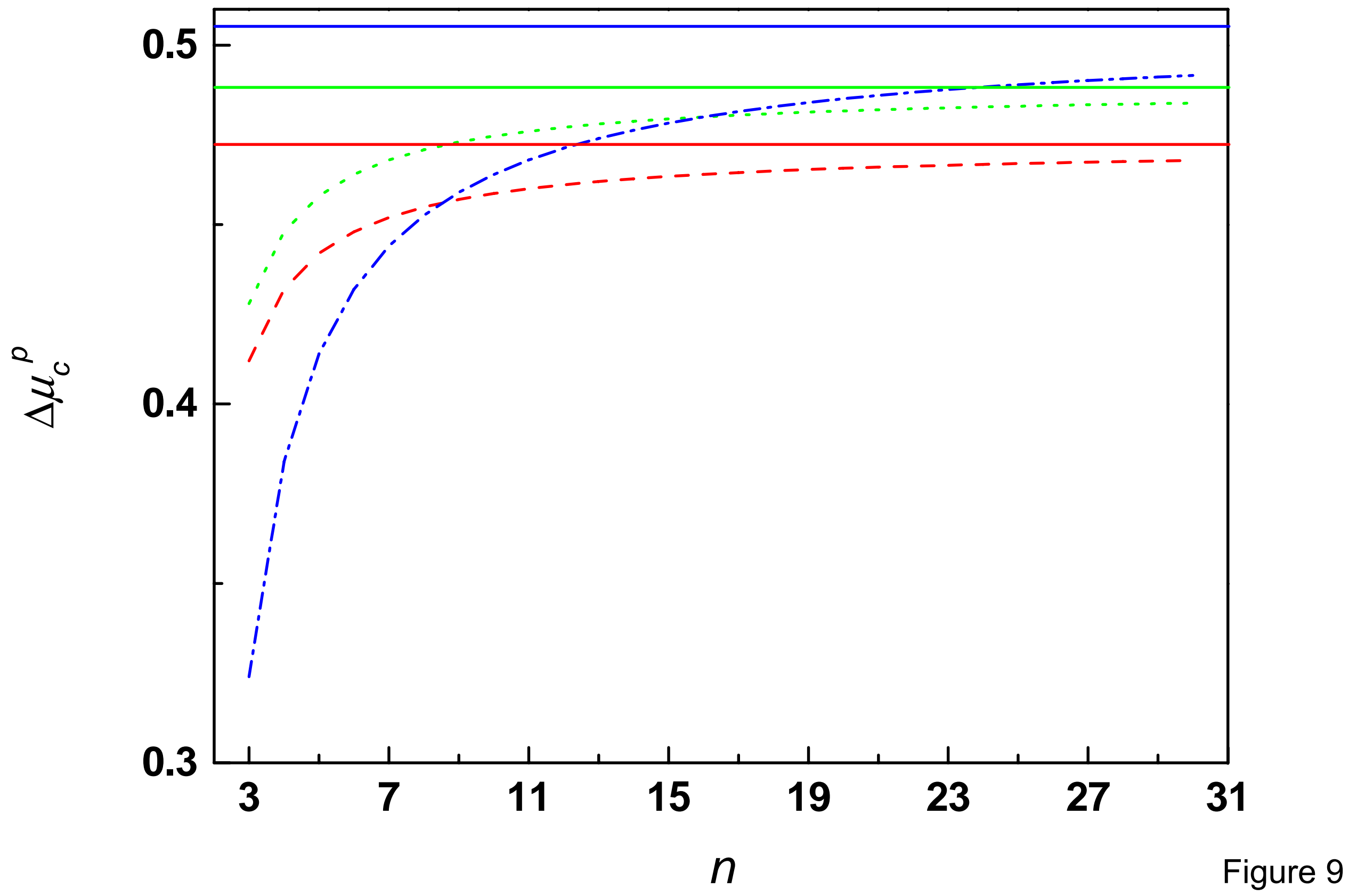




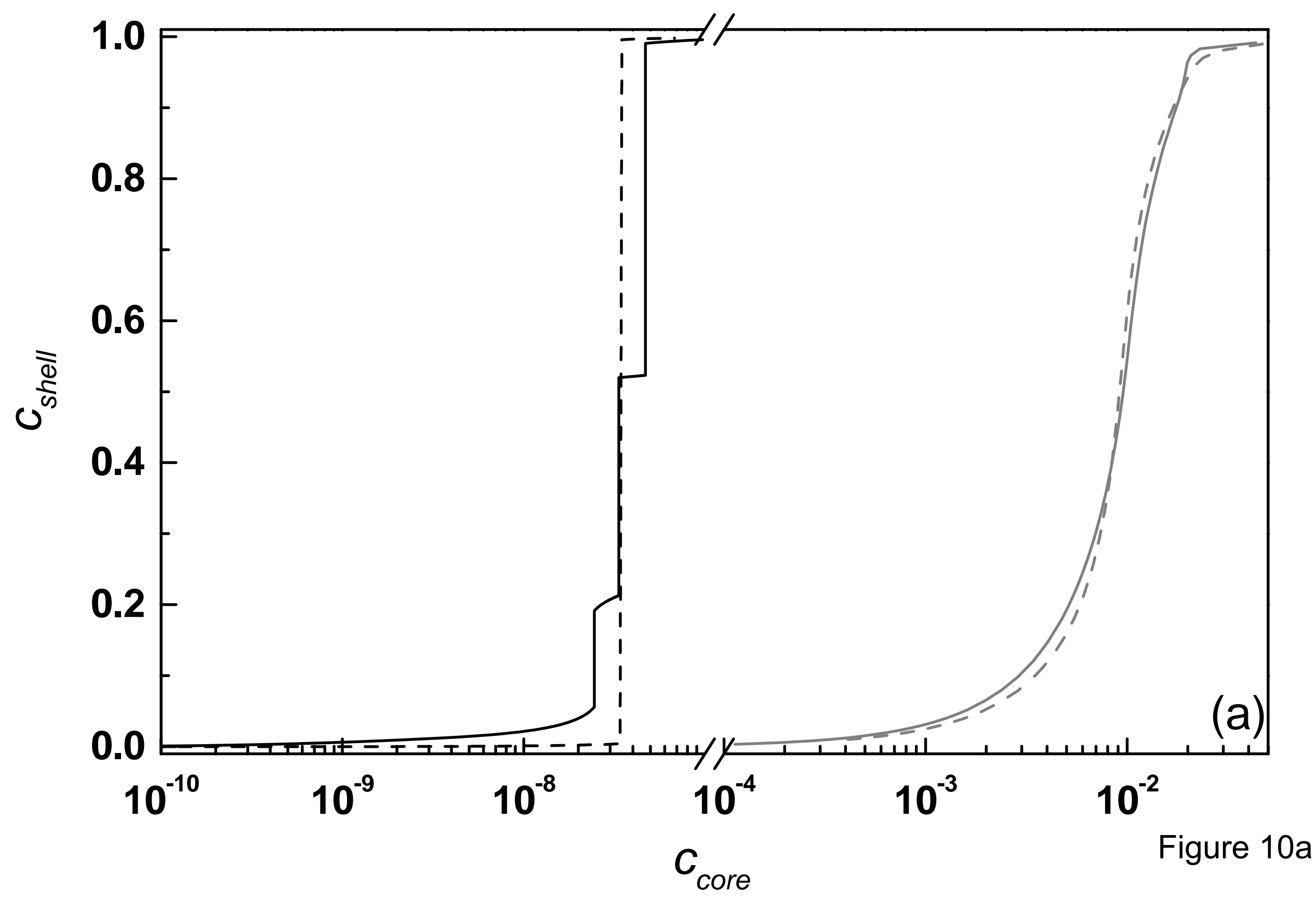




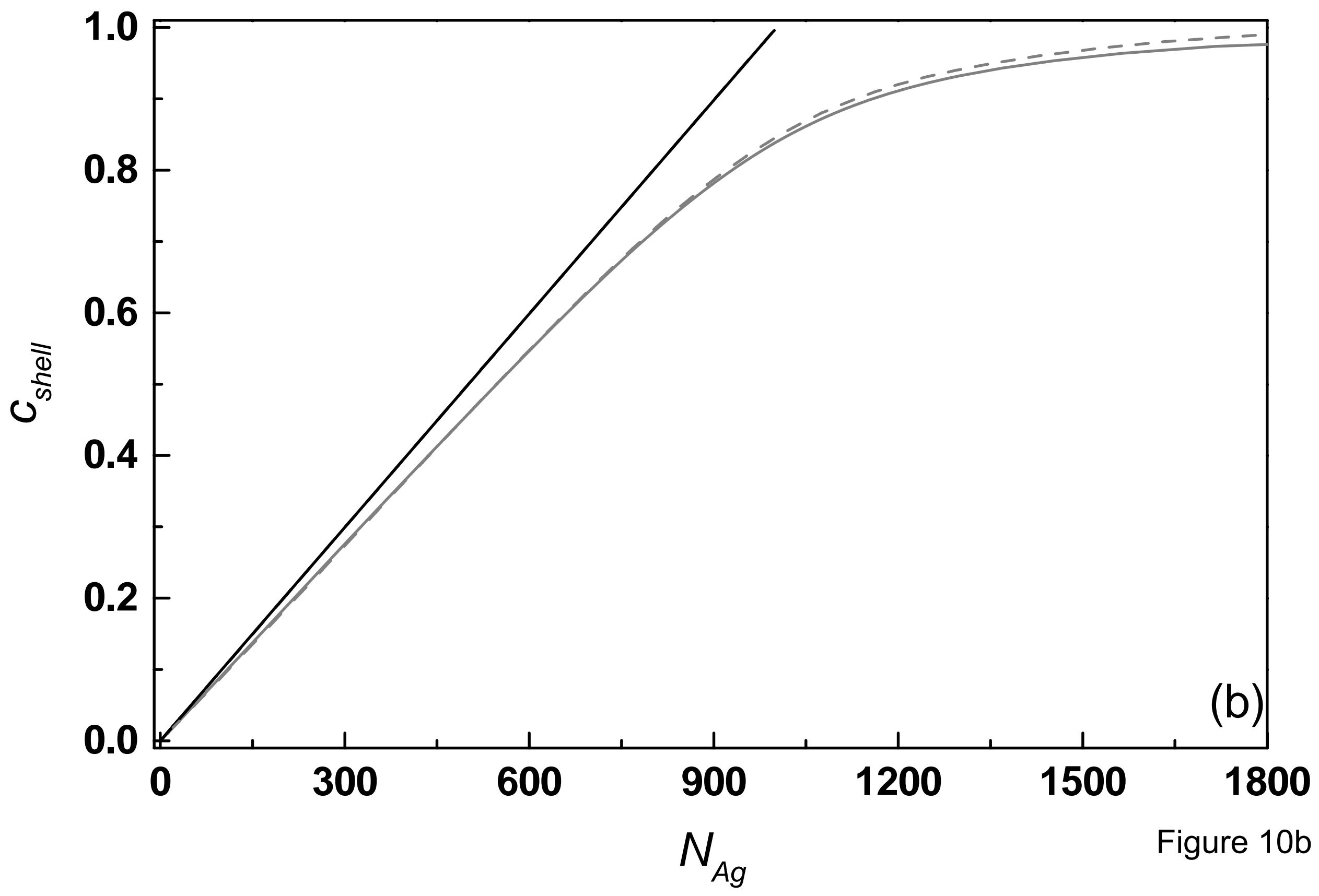

